\title{
High bandwidth RF photonic signal processing with Kerr micro-combs
}

\author{
David J. Moss
}

Optical Sciences Centre, Swinburne University of Technology, Hawthorn, VIC 3122, Australia. (dmoss@swin.edu.au).

\begin{abstract}
Integrated Kerr micro-combs, a powerful source of many wavelengths for photonic RF and microwave signal processing, are particularly useful for transversal filter systems. They have many advantages including a compact footprint, high versatility, large numbers of wavelengths, and wide bandwidths. We review recent progress on photonic RF and microwave high bandwidth temporal signal processing based on Kerr micro-combs with spacings from 49-200GHz. We cover integral and fractional Hilbert transforms, differentiators as well as integrators. The potential of optical micro-combs for $\mathrm{RF}$ photonic applications in functionality and ability to realize integrated solutions is also discussed.
\end{abstract}

Index Terms-Microwave photonics, micro-ring resonators.

\section{INTRODUCTION}

All-optical signal processing based on nonlinear optics has proven to be extremely powerful, particularly when implemented in photonic integrated circuits based on highly nonlinear materials such as silicon [1-3]. All optical signal processing functions include all-optical logic [4], demultiplexing at ultra-high bit rates from 160Gb/s [5] to over 1Tb/s [6], optical performance monitoring (OPM) using slow light [7,8], all-optical regeneration [9,10], and others [11-16]. Complementary metal oxide semiconductor (CMOS) compatible platforms are centrosymmetric and so the $2^{\text {rd }}$ order nonlinear response is zero. Hence, nonlinear devices in these platforms have been based on the $3^{\text {rd }}$ order nonlinear susceptibility including third harmonic generation [11,17-21] and the Kerr nonlinearity $\left(n_{2}\right)[1,2]$. The efficiency of Kerr nonlinearity based all-optical devices depends on the waveguide nonlinear parameter $(\gamma)$. Although integrated silicon nanowires can achieve extremely high nonlinear parameters $(\gamma)$, they suffer from high nonlinear optical loss due to two-photon absorption (TPA) and the ensuing free carriers [2]. Even with the use of p-i-n junctions to sweep out the carriers, the nonlinear figure of merit (FOM $=n_{2} /(\beta \lambda)$, where $\beta$ is the TPA and $\lambda$ the wavelength) of silicon is only 0.3 in the telecom band - too low to achieve good performance. While TPA can be used as a positive tool for some nolinear functions [22-24], for most processes the low FOM in the telecom band poses a problem. This has motivated research on other nonlinear optical platforms including chalcogenide glass [25-34]. However, although these platforms may offer advantages, most are not compatible with CMOS fabrication which is a significant consideration for practical and cost effective manufacturing.

Around 2007, new platforms for nonlinear optics were reported that are CMOS compatible with negligible TPA in the telecom band, including silicon nitride [35, 36] and high-index doped silica glass (Hydex) [37-47]. In addition to no nonlinear absorption, these platforms displayed a reasonable Kerr nonlinearity that yielded an extremely high nonlinear FOM as well as a nonlinear parameter high enough to sustain substantial parametric gain. Following the first report of a micro-ring resonator (MRR) based frequency comb source, driven by the Kerr optical nonlinearity in 2007 [48], the first fully integrated optical parametric oscillators were reported in 2010 [36, 37] in these new CMOS compatible platforms. Since 2010 the field of integrated micro-combs, or "Kerr combs" has become one of the largest fields in optics and photonics [47]. Integrated optical Kerr frequency comb sources, or "micro-combs" are a new and powerful tool to accomplish many new functions on an integrated chip, due to their very high coherence, while also offering flexible control wavelength spacing. Optical micro-combs are produced via optical parametric oscillation driven by modulational instability, or parametric, gain in integrated ring resonators, offering huge advantages over more conventional multiple wavelength sources. Many breakthroughs have been reported with Kerr micro-combs, from innovative mode-locked lasers [49-52] to quantum optical photonic chips [53-61], ultrahigh bandwidth optical fiber data transmission [62-64], optical neural networks [65-67], integrated optical frequency synthesizers [68]. Microcombs have been extensively reviewed [47, 69 - 76]. The success of these new CMOS platforms motivated the search 
for even higher performing CMOS compatible platforms including as amorphous silicon [77] and silicon rich silicon nitride [78], searching for the combination of low linear and nonlinear loss together with a high nonlinearity.

All-optical signal processing based on nonlinear optics has attracted significant interest over the years for its ability to achieve ultrahigh bandwidth operation without optical to electronic (or visa versa) conversion, for signal processing functions for telecommunications and RF/microwave systems. RF photonic applications include radar systems to signal generation and processing [79-128], and are attractive because of the ultra-high bandwidths as well as low transmission loss and strong immunity to electromagnetic interference. There are different RF photonic approaches including methods that map the optical filter response onto the RF domain. This is perhaps best represented by integrated devices based on stimulated Brillouin scattering [88-95], that have achieved high RF resolution — down to $32 \mathrm{MHz}$, a stopband discrimination $>55 \mathrm{~dB}$. A key approach to reconfigurable transfer functions for signal processing has been transversal filter methods [96-100]. These operate by generating progressively delayed and weighted replicas of an RF signal multicast onto many optical carriers, which are subsequently summed via photo-detection. Transverse filters can achieve a wide range of RF functions solely by varying the tap weights, and so this approach is very attractive for advanced dynamically adaptive RF filters. Discrete diode laser arrays [101] and fibre and integrated Bragg grating arrays and sampled gratings [103] have been used to generate the required taps. However, while offering advantages, these methods have increased complexity and footprint, limiting performance due to the limited number of wavelengths. Alternative methods such as electro-optic (EO) or acousto-optic (AO) combs [102,104,105], can help overcome this, but they require many high bandwidth modulators and high-frequency RF sources.

Kerr micro-combs were invented about 10 years ago [36, 37] and have been very successful. They provide advantages over other sources of multiple wavelengths for RF devices. They have achieved extremely high bandwidth data communications as well as a wide range of microwave signal processing devices [107-128]. Their combs spacings can be much wider than electro-optic combs, and in many ways EO and micro combs are complementary. EO combs excel at finer spacings from 10's of megahertz to $10-20 \mathrm{GHz}$, while integrated micro-combs typically have much wider spacings from 10's of GHz to 100's of GHz and even THz. Larger comb spacings have much wider Nyquist zones for large RF bandwidth operation, whereas smaller spacings provide many more wavelengths or RF "taps", although at the expense of a smaller Nyquist zones. Micro-combs provide more wavelengths while still with a large FSR, all in a small footprint. For RF transversal filters the number of wavelengths determines the number of channels for RF true time delays and RF filters [85, 121]. Systems such as beamforming devices [112] can also be improved in quality factor and angular resolution. Other approaches to filtering include RF bandwidth scaling [125] that yields a particular bandwidth for each wavelength channel, with the total bandwidth (maximum RF signal bandwidth) will depend on the number of wavelengths which is dramatically increased with micro-combs.

Recently [121], we reviewed transversal filtering and bandwidth scaling methods based on Kerr micro-combs, as applied to RF and microwave spectral filters. In this paper, we review microwave and RF high bandwidth temporal signal processing based on Kerr micro-combs. We cover both integral and fractional order Hilbert transformers and differentiators, as well as RF integrators. We discuss the trade-offs between wide spaced micro-combs with an FSR of $200 \mathrm{GHz}$ [109-111] with record low FSR micro-combs with a spacing of $49 \mathrm{GHz}$, operating via soliton crystals [122128]. We highlight their potential and future possibilities, contrasting the different methods and use of the differently spaced micro-combs. While $200 \mathrm{GHz}$ Kerr micro-combs have been successful for RF transversal filters, allowing high versatility and dynamic reconfigurability, their large comb spacing limits the number of wavelengths to typically $<20$ in the C-band. This is an important consideration because transversal filters need many components such as optical amplifiers and spectral shapers that are only available at telecom wavelengths $(1530-1620 \mathrm{~nm})$. This limitation in the number of wavelengths has limited the frequency resolution, bandwidth, and dynamic reconfigurability of micro-comb based RF filters. To overcome this, we focused on RF transversal filters with record high numbers of wavelengths up to 80 in the C-band [117]. This represents the highest for micro-comb based RF filters, and is a result of a record low spacing of $49 \mathrm{GHz}$ for the Kerr micro-comb. This resulted in RF filters with [121] $Q_{\mathrm{RF}}$ factors bandpass filters what were 4 times higher than the $200 \mathrm{GHz}$ combs yielded. In turn, for time dependent signal processing this results in a significant improvement in both reconfigurability and bandwidth. Our results confirm the feasibility of achieving high performance reconfigurable transversal RF filters for signal processing with reduced footprint, complexity, and cost. 


\section{INTEGRATED KERR MICRO-COMBS}

The formation of micro-combs is complex and arises from a combination of a high nonlinear parameter together with low nonlinear and linear loss, and finally with careful dispersion engineering. Many different material platforms have been reported for micro-combs [47] including silica, magnesium fluoride, silicon nitride, and doped silica glass [47, 70, 85]. In 2008 [39] we reported efficient FWM at low (milliwatt) powers with a 575GHz FSR spaced comb, generated by a ring resonator with a relatively low Q-factor of about 60,000. This was the first report of low power continuous wave (CW) nonlinear optics in a silica glass based platform, and in 2010 was followed by the first report of an integrated micro-comb [37, 38]. These were realized in Hydex and silicon nitride and were inspired by the first micro-combs in toroid resonators [48]. A significant development came [116, 117] with integrated micro-combs having record low FSR's of $<50 \mathrm{GHz}$, significantly increasing the number of available wavelengths to over 80 in the telecom band. Apart from their low spacing, these micro-combs operated via a different process than DKS states [66-73], a process termed soliton crystals [129, 130]. Many breakthroughs have been achieved with micro-combs, including ultralow pump power combs [131], dark solitons [132], laser-cavity solitons [133] and others [134-139].

The microcombs that formed the basis of the research discussed here were fabricated in Hydex glass [37, 38], a CMOS compatible platform. Ring resonators with Q's from 60,000 to greater than 1.5 million have been reported, with radii from 592 - $48 \mu \mathrm{m}$, corresponding to FSRs from 49 - $575 \mathrm{GHz}$. The RF signal processors reviewed here were based on combs with spacings of $200 \mathrm{GHz}$ (Fig. 1a) and $49 \mathrm{GHz}$ (Fig. 1b). Hydex glass layers $(n=\sim 1.7 \mathrm{at} 1550 \mathrm{~nm})$ were deposited via PECVD (plasma enhanced chemical vapour deposition), and patterned via deep UV stepper mask aligner photolithography. The waveguides were reactive ion etched to create waveguides with very low surface roughness. An upper cladding layer of silica glass $(n=\sim 1.44$ at $1550 \mathrm{~nm})$ was then deposited. We typically use a vertical coupling scheme between the bus and ring resonator, with a gap of about 200nm that can be controlled by film growth - more accurate than lithography. The advantages Hydex include its very low linear loss $\left(\sim 0.06 \mathrm{~dB} \cdot \mathrm{cm}^{-1}\right)$, its reasonable nonlinear parameter $\left(0.233 \mathrm{~W}^{-1} \cdot \mathrm{m}^{-1}\right)$, and negligible TPA even up to many $\mathrm{GW} \cdot \mathrm{cm}^{-2}$. We reported high Q's 1.5 million (Fig. 1) for both the 49GHz and 200GHz FSR MRRs. After packaging with fiber pigtails, the coupling loss was about $0.5-1 \mathrm{~dB}$ per facet, achieved with on-chip mode converters.

To generate combs with the $200 \mathrm{GHz}$ devices, the $\mathrm{CW}$ pump power was amplified to $>+30 \mathrm{dBm}$ and the wavelength was tuned from blue to red near a TE resonances at $\sim 1550 \mathrm{~nm}$. When the difference between the pump and cold resonance wavelength became sufficiently small it resulted in the intracavity power reaching threshold. At this stage, modulation instability gain resulted in oscillation [47], generating primary combs with a spacing given by the MI gain peak - a function of the dispersion and intra-cavity power. As the detuning was varied, single FSR spaced microcombs appeared. While these states were not solitons, we found that operating in single soliton states such as the dissipative Kerr solitons (DKS) [66] was in fact not necessary. This is significant since, while much is now understood about DKS solitons and significant progress has been made [139], DKS states require complicated pump tuning dynamics with simultaneous amplitude and wavelength sweeping, including in reverse directions, to be able to "kick" the solitons out of chaotic states. Our early work on micro-combs was based on the $200 \mathrm{GHz}$ FSR combs that operated in this partial coherence. While not rigorous soliton states, they were still low noise and avoided the chaotic regime [47] - we found them to be sufficient for our RF work. While their spectra (Figs. 1b,c) indicate that they are not solitons, nonetheless they successfully demonstrated many functions for RF signal processing [85, 107, 109 -111].

More recently, we have used microcombs featuring a record low spacing of $49 \mathrm{GHz}$, based on soliton crystals [129, 130]. These arise from mode crossings and are easier and more robust to generate than other solitons, and even partially coherent states. They can be generated without complex pump dynamics, and indeed can be achieved even via manual control of the pump wavelength. The reason for this lies in the fact that the internal optical energy in the cavity of the soliton crystals is very close to the chaotic state, and so when they are generated from chaos there is only a small change in optical cavity energy, resulting in almost no shift in resonant wavelength. It is the shift in resonant wavelength and internal energy that makes DKS states challenging to generate. This also greatly increases the efficiency of the soliton crystals (comb line relative to the pump energy) relative to DKS states (single solitons in particular). However, the compromise with soliton crystals is that their spectra are not flat - they have nonuniform "curtain" patterns. While this can require spectral flattening, it has not prevented them from achieving many high performance microwave and RF functions. Soliton crystals are based on a mode-crossing in the resonator and so this needs to be engineered, as well as anomalous dispersion, but this has not posed a significant difficulty and high fabrication yield is achievable [64]. 
When generating soliton crystals, we typically tuned the pump laser wavelength manually across a resonance (TE polarized). When the pump (with sufficient power) was aligned well enough with the resonance, a primary comb was generated, similar to the $200 \mathrm{GHz}$ states. In this case, however, as the detuning was changed further, distinctive 'fingerprint' optical spectra were observed (Fig. 2), arising from spectral interference between tightly packed solitons in the cavity - solitons that formed the soliton crystals $[129,130]$. We observed a small abrupt jump in the intracavity power (Fig. 2(b)) when these spectra appeared. At the same time we observed a large reduction in the RF intensity noise (Fig. 2(c)). These observations indicate soliton crystal formation [38], although to prove this one would need to perform time-resolved autocorrelations. The specific micro-comb states reached was not important for RF applications, only that low RF noise and high coherence be achieved, and we found this to be easy to generate with simple pump wavelength tuning. Soliton crystals yielded the lowest noise of all micro-comb states, and we have focused on these for our microcomb work, including a low phase-noise microwave oscillator [120].

\section{RF TRANSVERSAL FILTER: THEORY AND EXPERIMENT}

The transfer function of a general transversal structure can be described as [85]

$$
F(\omega)=\sum_{k=0}^{M-1} h_{k} e^{-j \omega K T}
$$

Where $j=\sqrt{-1}, \omega$ is the angular frequency of the RF signal, $M$ is the tap number, $T$ is the time delay between taps, $h_{k}$ is the $k_{t h}$ tap coefficient for the discrete impulse response of $F(\omega)$, obtained performing the inverse Fourier transform of $F(\omega)$, temporally windowed with a cosine bell. The transversal filter is equivalent to a finite impulse response digital filter. Both are fundamental tools for RF signal processing. By designing the tap weights $h_{k}$ for each wavelength, different signal processing transfer functions can be obtained including integrators, differentiators, Hilbert transforms and more. The response function Nyquist frequency (maximum RF frequency) is given by $f_{\text {Nyquist }}=1 / 2 T$. Figure 3 shows the transversal filter and multiple wavelength source. First, the RF signal is multicast onto all microcomb wavelengths with a modulator, followed by transmission through a dispersive device to generate a wavelengthdependent delay. Second-order dispersion, yielding a linear relationship between wavelength and delay, is used to progressively delay the replicas. These delayed signals can be either separately converted into electronic RF signals with wavelength demultiplexers and photodetector arrays for applications such as RF true time delays [116], or summed by photodetection for RF transversal filters signal processing [85]. The large number of wavelengths from the microcomb has major advantages since the number and amplitude of progressively delayed RF replicas, or taps, determines the performance of the system. By setting the tap coefficients, a reconfigurable transversal filter with virtually any transfer function can be realized, including both integral and fractional differentiators, [109,127] Hilbert transformers [107, 126], integrators [123], waveform generators, [128] bandpass filters [111,117, 121], and more.

Figure 4 shows the transversal filters for the $200 \mathrm{GHz}$ (Fig 4a) and $49 \mathrm{GHz}$ (Fig. 4(b)) combs. In both cases the combs are amplified and then processed by one $(200 \mathrm{GHz}$ case) or two ( $49 \mathrm{GHz}$ case) waveshapers to flatten then shape the spectra. A second waveshaper was used for the 49GHz FSR comb to pre-flatten the spectra of the soliton crystals. The power of each comb line was controlled by the waveshaper according to the tap weights. To increase the accuracy, real-time feedback control was used to shape the comb line power. The comb lines were then spatially divided into two according to the sign of the tap coefficients - one path for negative weights and one for positive. Next, for the $200 \mathrm{GHz}$ micro-comb experiments the signal was passed through a $2 \times 2$ balanced Mach-Zehnder modulator (MZM) biased at quadrature which simultaneously modulated the input RF signal on both positive and negative slopes, yielding replicas of the signal with phase and tap coefficients having both signs. For 49GHz FSR experiments, both positive and negative taps were achieved by spatially separating the wavelengths according to the tap coefficients and then feeding them into the positive and negative input ports of a balanced photodetector (Finisar BPDV2150R) (Figure 4b).

For the $200 \mathrm{GHz}$ experiments, the signal was modulated by the MZM and went through $\sim 2.122-\mathrm{km}$ of standard single mode fibre (SMF, $\sim 17.4 \mathrm{ps} / \mathrm{nm} / \mathrm{km}$ ), yielding a delay $T \sim 59 \mathrm{ps}$ between wavelengths, or taps (the channel spacing of the time delay lines equalled the micro-comb FSR), resulting in a Nyquist frequency of $\sim 8.45 \mathrm{GHz}$. The bandwidth of the systems (Nyquist frequency) could be increased by decreasing the time delay which, because of the large FSR of the compact MRR, could reach beyond $100 \mathrm{GHz}$. Finally, the weighted and delayed taps were combined by a highspeed photodetector (Finisar, $40 \mathrm{GHz}$ bandwidth) into electronic RF signals. 
For the 49GHz FSR microcomb based transversal filter, the signal went through $5 \mathrm{~km}$ of SMF to generate the delay taps. The fibre dispersion was identical $(17 \mathrm{ps} / \mathrm{nm} / \mathrm{km})$ in the $200 \mathrm{GHz}$ system, but the time delay between adjacent taps was different at $T \sim 34.8 \mathrm{ps}$. This resulted in a bandwidth (Nyquist frequency, half of $F S R_{\mathrm{RF}}$ ) of $14.36 \mathrm{GHz}$. This can be increased by decreasing the time delay (with shorter fiber), although with reducing the tuning resolution. The transversal filter bandwidth was limited by the comb spacing. For the $49 \mathrm{GHz}$ comb, significant crosstalk between adjacent wavelength channels occurred for RF frequencies $>24.5 \mathrm{GHz}$. This could be remedied by using a micro-comb with a larger spacing, although at the expense of yielding fewer comb lines over the C-band. Although standard optical fibre is often used to generate the delays, this can be done more efficiently with other methods. Typically, only $2-\mathrm{km}$ of SMF, with a dispersion of $34 \mathrm{ps} / \mathrm{nm}$, is needed. This is within the range of multi-channel tunable dispersion compensators [140-143], which can also be designed with a built-in dispersion slope offset. This would not only yield a compact and latency free delay but enable tunability to adjust the Nyquist zone. In the following sections, we review progress made in high bandwidth temporal RF and microwave signal processing functions beginning with Hilbert transforms, followed by differentiation and then integration, all based on Kerr micro-combs.

\section{HILBERT TRANSFORMS}

The Hilbert transform (HT) is a fundamental signal processing operation with wide applications to single sideband modulators, radar systems, signal sampling, speech processing, measurement systems, and more [144]. Integral HTs perform a $\pm 90^{\circ}$ phase shift around a central frequency, with an all-pass amplitude transmission. Fractional Hilbert transforms (FHTs) that yield a variable phase shift represent a powerful extension to the standard HT, with applications to secure single sideband communications [145], hardware keys [145, 146], and in forming images that are edge enhanced relative to the input object, where one can select the edges that are enhanced as well as the degree of edge enhancement [147]. Electronic fractional Hilbert transformers are limited in bandwidth [145, 148], while photonic approaches yield wide bandwidths and a high level of EMI immunity. HTs using free-space optics [146, 149] have been reported that have yielded high levels of performance but these devices are complicated and large in size. Phaseshifted fibre Bragg grating based Hilbert transformers typically have bandwidths of a few 100GHz [150-153] but only yield a precise FHT for signals with fixed fractional orders, specific bandwidths, and do not operate on the RF signal itself but the complex optical field. This also holds for integrated reconfigurable microwave processors [154] as well Bragg grating approaches [155]. In practice, it is not the operation on the complex optical field that is of interest for RF signal reshaping and measurement but the operation on the actual RF and microwave signals [156-163].

Integrated Hilbert transformers realized using micro-ring or micro-disc resonators, Bragg gratings in silicon, and integrated InP-InGaAsP photonic chips yield compact devices with high stability and mass-producibility [154, 155, 159-161]. Nevertheless, they only operate on the complex optical field and generally only provide a limited phase shift tuning range. Transversal filter approaches offer high reconfigurability $[162,163]$ but need many discrete lasers, thus increasing system cost, complexity, and size, and restricting the tap number and hence performance. Optical frequency combs provide a single high-quality source that can generate many wavelengths. These include mode-locked lasers of various types [164], modulators [165], as well as resonators [166]. Of these, Kerr micro-combs generated by microresonators provide many wavelengths with greatly reduced footprint.

A fractional Hilbert transformer has a transfer function in the spectral domain given by [80, 126]:

$$
H_{P}(\omega)=\left\{\begin{array}{c}
e^{-j \varphi}, \text { if } 0 \leq \omega<\pi \\
e^{j \varphi}, \text { if }-\pi \leq \omega<0
\end{array}\right.
$$

where $j=\sqrt{-1}, \varphi=P \times \pi / 2$ is the phase shift, and $P$ denotes the fractional order. From Eq. (2), a FHT can be viewed as essentially a phase shifter with a phase shift of $\pm \varphi$ centered about a frequency $\omega$. This defaults to a standard integral HT when $P=1$. The impulse response is a continuous hyperbolic function:

$$
h_{P}(t)=\left\{\begin{array}{c}
\frac{1}{\pi t}, t \neq 0 \\
\cot (\varphi), t=0
\end{array}\right.
$$

For digital transforms, this hyperbolic function gets truncated and temporally sampled with the discrete taps. The sample time step $\Delta t$ yields the null frequency $f_{\mathrm{c}}=1 / \Delta t$. The order of the FHT can be tuned continuously by only varying the tap coefficient at $t=0$ without varying any of the other tap coefficients [146]. 
To achieve a Hilbert Transform the normalized power of each comb line is given by:

$$
p_{n}=\frac{1}{\pi|n-N / 2+0.5|}
$$

where $N$ is the number of or filter taps or comb lines, and $n=0,1,2, \ldots, N-1$ is the comb index.

In 2015, the first Kerr micro-comb based Hilbert transformers was demonstrated, that employed as many as 20 wavelengths [107] from a microcomb that had an FSR of 200GHz. This device achieved a record-high RF range of 5 octaves. Figures 5(a) and 5(b) show the shaped optical micro-combs measured with an optical spectrum analyzer for combs having 20 and 12 wavelengths, respectively. The designed wavelength target powers, or tap weights, are shown (green crosses) in Figs. 5(a) and 5(b). The waveshaper shaped the comb line powers to within $+/-0.5 \mathrm{~dB}$ of the designed weights. This was feasible since the waveshaper had a resolution of $10 \mathrm{GHz}$ much less than the comb-line spacing of $200 \mathrm{GHz}$. After the comb line powers were adjusted to give the correct weights needed for the HT impulse response, with the un-needed comb lines extinguished below the noise floor, the RF frequency amplitude and phase response was measured using a vector network analyzer (VNA).

Figure 6a shows the photonic HT filter RF amplitude response in frequency for 12, 16 and 20 taps. All measurements agree with theory, showing very low $(<3 \mathrm{~dB})$ amplitude ripple. All 3 filters were measured to have the same null frequency at $16.9 \mathrm{GHz}$, corresponding a tap spacing of $\Delta t=1 / f_{c}=59 \mathrm{ps}$, matching the delay difference between comb lines. This difference was equal to the $F S R$ of $1.6 \mathrm{~nm}$, and was generated after being transmitted through spool of 2.122-km SMF with a dispersion of $D=17.4 \mathrm{ps} / \mathrm{nm} / \mathrm{km}$. The null frequency could be adjusted by employing different lengths of fiber to adjust the delay tap spacing, or alternatively by using tunable dispersion devices [140-143]. Increasing the tap number increases the filter bandwidth so that with 20-taps, the Hilbert transformer had a 3-dB bandwidth from 0.3 - $16.4 \mathrm{GHz}$ - more than five octaves - which was a record for any HTs. The bandwidth can be increased even further using more comb lines. Indeed, only a small fraction of the generated comb lines was actually employed for these filters. The number of taps was actually limited by the waveshaper, which was a C-band device, as well as the EDFA bandwidth. Amplitude ripple over the passband can be reduced by apodizing the tap coefficients. Figure 6(b) shows the measured phase response as a function of the number of taps, showing a very similar response. Each shows a near constant phase close to $-90^{\circ}$ over the passband. There are some small variations compared with the ideal $-90^{\circ}$ phase near zero frequency, and particularly for the exact null frequency $f_{c}=16.9 \mathrm{GHz}$.

Recently, [126] Tan et. al used a Kerr microcomb having a much lower comb FSR of 49GHz that provided many more lines (80) across the $\mathrm{C}$ band, as the basis of a fractional Hilbert transformer (FHT). FHTs were achieved with phase shifts of $15^{\circ}, 30^{\circ}, 45^{\circ}, 60^{\circ}, 75^{\circ}$, and $90^{\circ}$, equivalent to fractional orders $(0.17,0.33,0.5,0.67,0.83,1.0)$ by shaping the comb lines with the required weights,. Up to 17 wavelengths were used - the space between the 8-9th and 9-10th lines was $0.8 \mathrm{~nm}$, with the remaining being $1.6 \mathrm{~nm}$, chosen to maximize the Nyquist zone. A 2.1-km length of single mode fibre $(\beta=\sim 17.4 \mathrm{ps} / \mathrm{nm} / \mathrm{km})$ provided a delay line between channels of $\tau=\mathrm{L} \times \beta \times \Delta \lambda=\sim 29.4 \mathrm{ps}$, consistent with a FSR $\mathrm{RF}_{\mathrm{RF}}$ of $1 / 2 \tau=\sim 17 \mathrm{GHz}$. This bandwidth can be varied (increased) by changing the length (shortening) of fibre.

The theoretical amplitude and phase response of the FHTs for all phase shifts are given in Figs. 7 (a)-(f) versus tap number. Fig. 8(a) and (b) show the RF frequency phase and amplitude response of a FHT with phase shift $45^{\circ}$ and 5 , 9, 13 and 17 taps. Figure 8c) shows the bandwidth (3dB) vs tap number where it is seen that the bandwidth increases with tap number. With 17 taps, the base-band RF bandwidth was $480 \mathrm{MHz}$ to $16.45 \mathrm{GHz}$ - more than 5 octaves - with a very small variation of $\pm 0.07 \mathrm{rad}$ in the phase within the band - a record performance for FHTs. For a standard $90^{\circ}$ HT the micro-comb generated $>40$ taps, reducing the amplitude ripple and root-mean-squared error (RMSE) across the passband [126]. Fig. 8 (c) shows that the theoretical bandwidth rapidly increases with tap number to 17, after which it levels off, and so 17 - 20 is the optimum tap number.

Figure 9 (ii)-(iii) shows theory and experimental results for the FHT with orders from $0.166-0.833$ (phase shift $15^{\circ}$ $-90^{\circ}$ ). The FHT normalized magnitude and phase frequency response and temporal response (Fig. (iv)) are shown. The amplitude variation is $<+/-1.5 \mathrm{~dB}$ from $480 \mathrm{MHz}$ to $16.45 \mathrm{GHz}$ with phase variation $< \pm 0.07 \mathrm{rad}$ within the passband. Experiments for real-time FHTs with Gaussian pulses were also done. Fig. 9 (iv) shows a pulse after processing by the FHT together with theory. The resulting parameters are shown in Table I of [126]. We see that the experimental curves match the theory with good performance.

The more recent FHT results with soliton crystals and 49-GHz FSR yield much better results than the earlier 200- 
GHz HT in bandwidth because of the larger tap number. However, it is not always true that smaller FSRs are better. The 200-GHz devices can achieve extremely high RF bandwidths of $100 \mathrm{GHz}$ whereas $49 \mathrm{GHz}$ devices are restricted to $\sim 25 \mathrm{GHz}$. In summary, our results confirm that microcombs are very effective for high-bandwidth reconfigurable HTs and FHTs with reduced footprint, complexity, and cost.

\section{DIFFERENTIATION}

The fundamental operation of differentiation is a key function in RF and microwave signal processing. It has wide applications for RF spectral analysis, ultra-wideband (UWB) generation, and RF spectral filters [167-171]. Integral RF differentiators using photonic techniques [172-184] including self and cross-phase modulation (SPM, XPM) [172,173] [177-179] methods, and cross-gain modulation in semiconductor optical amplifiers (SOA) [176] have all been reported. Fractional differentiators (FDs) have unique capabilities over integral differentiators [127,182,183] and have played important roles in electricity and electronics, chemistry, biology, mechanics, and even economics. Their most wellknown applications have been mechatronics, control theory, and particularly image edge detection [182,183]. Despite these many applications, photonic fractional differentiators have received comparatively little attention.

Both fractional and integral photonic differentiators have centred around differentiation of the complex optical field, as opposed to the RF signal. Although successful, a photonic differentiator using a dual-drive MZM with an RF delay line [174] was limited in speed by the RF delay line bandwidth, and while those based on optical filters [175] have featured speeds up to 40-Gb/s, they typically have a fixed integral differentiation order and lack reconfigurability and flexibility. Transversal schemes for highly reconfigurable differentiators using discrete laser arrays $[169,180,181]$ are a powerful approach, but also have limitations including in the number of wavelengths. Increasing the number of lasers increases the system complexity, cost, and size, and limits the performance. A single source that can generate many wavelengths would be highly advantageous. A photonic RF integral differentiator with 8 taps [109] using a 200-GHz FSR micro-comb source recently achieved $1^{\text {st }}, 2^{\text {nd }}$, and $3^{\text {rd }}$ order derivatives at RF bandwidths up to $17 \mathrm{GHz}$, with the potential to reach $100 \mathrm{GHz}$.

A temporal fractional $N_{\text {th }}$-order differentiator is a linear and time-invariant system with transfer function [168]

$$
H_{N}(\omega) \propto(j \omega)^{N}
$$

where $j=\sqrt{-1}, \omega$ is the angular RF frequency, and $N$ is the fractional or even complex $[184,185]$ order of the derivative. According to Eq. 5, the temporal differentiator amplitude response is proportional to $|\omega|^{N}$, versus the phase response that is linear with a phase step of $N \pi$ at the null frequency.

Transverse filter based photonic RF differentiators have a finite set of weighted and delayed replicas of the RF signal in the optical domain that are combined by photodetection. The transfer function (Eq. (5)) is

$$
H_{N}(\omega)=\sum_{n=0}^{M-1} a_{n} e^{-j \omega n T}
$$

where $a_{n}$ is the tap coefficient of the $n^{\text {th }}$ tap, $T$ is the delay time between taps and $M$ is the tap number. Differentiators described by Eq. (6) are RF intensity differentiators where the output signal after detection is a derivative of the RF signal, in stark contrast to optical field differentiators that generate the complex optical field derivative [167,171,153,154,185-187].

Recently, [109] the first RF differentiator based on a micro-comb was reported, achieving integral $1^{\text {st }}, 2^{\text {nd }}$, and $3^{\text {rd }}$ order derivatives of the RF signal. That device was based on a 200GHz spaced Kerr micro-comb with 8, 6 , and 6 taps and had a dynamic range of $\sim 30 \mathrm{~dB}$. More taps would be needed to increase the differentiation order, so that increasing the order of differentiation for a fixed number of taps also increases the required power dynamic range. To obtain better performance for a fixed number of taps, the bandwidth of the integral differentiators was decreased to $1 / 2$ the Nyquist frequency. To implement Eq. (5) for a temporal differentiator, tap weights (Eq. (6)) were calculated with the Remez algorithm [188] and are listed in Table I of [109] for $1^{\text {st }}, 2^{\text {nd }}$, and $3^{\text {rd }}$ order derivatives. For other RF functions, the accuracy of the comb shaping was improved via real-time feedback control. The phase and amplitude response of $1^{\text {st }}$, $2^{\text {nd }}$, and $3^{\text {rd }}$ order differentiators are plot in Figs. 10 (a)-(c) versus tap number. As the tap number increases, the error between the perfect and actual theoretical amplitude response is improved for all orders. In contrast, the phase response is the same as the ideal differentiator independent of the tap number.

The shaped optical comb spectra shown in Figs. 11 (b)-(d) reveal a good match between the measured comb lines' power (red solid line) and the calculated ideal tap weights (green crosses). Figures 12 (a-i), (b-i), and (c-i) show the measured and calculated amplitude response of the differentiators. The corresponding phase response is depicted in 
Figs. 12 (a-ii), (b-ii), and (c-ii) where it is clear that all three differentiators agree well with theory. The periodicity of the RF response was $\sim 16.9 \mathrm{GHz}$, given by the delay time between taps. By adjusting this periodicity by programming tap coefficients changing the fibre delay, a variable bandwidth can be achieved, which is useful for different applications. The ability to change the derivative order just by changing tap weights is a powerful method reconfigure the system functionality dynamically without new hardware, and is not available with other approaches such as optical filters.

Real-time differentiators have also been achieved for 120 ps pulses (Fig. 13 (a)) shown in Figs. 13 (b)-(d) for different orders, along with theory, showing good agreement. Unlike optical field differentiators $[167,171,153,154,185-187]$, RF intensity derivatives are achieved for $1^{\text {st }}, 2^{\text {nd }}$, and $3^{\text {rd }}$ order differentiators with RMSE's of $\sim 4.15 \%, \sim 6.38 \%$, and $\sim 7.24 \%$, respectively.

Tan et.al recently [127] achieved the first photonic fractional-order RF differentiator operating on the RF signal and not the complex optical field, using a 49-GHz-FSR Kerr micro-comb that generated a large number of wavelengths ( 80 in the $\mathrm{C}$ band), yielding a wide bandwidth of $15.49 \mathrm{GHz}$. By shaping the comb lines with the required weights, dynamically changeable fractional orders from 0.15 - 0.9 were achieved. Real-time fractional differentiation of input pulses were also performed, achieving good agreement with theory. This confirms this an effective way of implementing reconfigurable high-speed fractional differentiators with reduced complexity, footprint, and even possibly cost.

The success of the differentiator was due to the soliton crystals as well as a low $48.9 \mathrm{GHz}$ FSR, that together provided $>80$ wavelengths over the C-band. The system performance was helped by the soliton crystal's robust and stable operation, easy initiation and its high efficiency. The relationship between the tap number and performance was also investigated. Figure 14 shows the theoretical fractional order differentiator transfer function for 6 orders and different tap numbers, along with theory. As seen, the RF differentiator bandwidth agree well with the agreement getting better with increasing number of taps. Figure 15 (a) shows that the tap number has a large effect on the fractional differentiator bandwidth, as seen in the experiments for a fractional order of 0.45 (Figs. 15 (b) and (c)). Increasing the number of taps to 27 increased the bandwidth to $15.49 \mathrm{GHz},>91 \%$ of the Nyquist band. As many as 29 wavelengths were experimentally used for the taps, which can be increased by improving the optical signal-to-noise ratio limited by the optical amplifier noise.

The comb spectra, shaped to produce the derivatives (Fig. 16), show that the optical power for each comb line agrees well with the designed tap weights. The fractional differentiator frequency response was measured with a vector network analyzer, showing that the power response had a bandwidth of DC to $15.49 \mathrm{GHz}$. The achieved range of fractional orders (Fig. 17) resulted from the close agreement between experiment and theory for the slope coefficients of the power response and shift in the phase response. Figure 18 shows the temporal response of the fractional differentiator for all orders, using a 200 ps wide RF Gaussian pulse (Fig. 18) generated by an arbitrary waveform generator. The good agreement with theory indicates the achievable range of orders that are possible with our approach to fractional differentiators.

\section{INTEGRATION}

Temporal integration is a key capability for signal processing systems and photonic based methods offer many advantages including wider bandwidths than electrical integrators along with a strong EMI immunity and low loss [79,80,189]. Many photonic integrators have been reported including grating [190-192] and micro-ring resonator $[15,42,193]$ based approaches. These have achieved time resolutions as short as 8 ps [193] with large time-bandwidth products that result from very high Q factor resonantors. These approaches, however, are still limited in many ways, including the fact that their parameters can't be varied dynamically including the time resolution or integration window, limiting their ability to process different RF signals. More significantly, these operate on the optical field, not RF signals themselves unless they have electrical to optical conversion which limits the bandwidth. Another important approach uses transversal filters that offer accuracy and reconfigurability resulting from parallelism, where each path is independently controlled [194-196]. By tailoring the delay, RF integration with a variable bandwidth can be realized [194-196]. Still, these have limitations due to the limited channel number for electro-optic combs or diode laser arrays, for example. These approaches have trade-offs between wavelength number and system complexity and ultimately limit the number of channels, in turn restricting the time-bandwidth product.

Kerr optical micro-combs in integrated platforms [38, 47, 68-70,118,197] offer advantages for RF integration, including high wavelength numbers and reduced footprint and complexity. Recently, a reconfigurable temporal RF 
photonic integrator was reported using a soliton crystal micro-comb [123] where the RF signal was multicast onto the comb lines, progressively delayed and then combined via photodetection. The large number of wavelengths (81) in that work yielded a large time integration window of $\sim 6.8 \mathrm{~ns}$ and resolution of $\sim 84 \mathrm{ps}$.

Figure 19 shows the transversal filter photonic RF integrator operation principle, where integration is achieved by a discrete convolution time-spectrum operation between the RF signal $f(t)$ and the micro-comb, described by:

$$
y(t)=\sum_{k=1}^{N} f(t+k \cdot \Delta t)
$$

where $N$ is the number of wavelengths and the delay step $\Delta t, j=\sqrt{-1}$. After the $f(t)$ replicas are progressively delayed and combined, the integration of $f(t)$ results with a time resolution of [194] $\Delta t$, an bandwidth of $1 / \Delta t$, and integration time window $T=N \times \Delta t$. The transmission frequency response of a continuous, or non-discrete, ideal integrator is linear with $1 /(j \omega)$, where $\omega$ is the angular frequency, and is a low pass sinc filter [111,117], given by

$$
H(\omega)=\sum_{n=0}^{N-1} e^{-j \omega n \Delta t}
$$

The experiment for the RF photonic integrator is similar to Figure 4, but based on the $49 \mathrm{GHz}$ soliton crystal microcomb that featured very low intensity noise - it has even been used as a local oscillator [120]. Thus, the noise of the micro-comb did not deteriorate the integrator noise performance. The RF signal was multicast onto the micro-comb lines flattened by a WaveShaper via an electro-optical modulator (EOM). The replicas were progressively delayed via transmission through $13 \mathrm{~km}$ of standard optical fibre and summed with a high-speed photodetector. The adjacent wavelength delay $(\Delta t)$ was given by the dispersion and length of the fibre and spacing between comb lines, and this determined the integrator resolution [194]. The temporal resolution was $\sim 84$ ps which can be made arbitrarily small by reducing the delay line dispersion, although with a decrease in the integration window. Sixty lines in the C-band were used, resulting in a time window $(T=N \times \Delta t)$ of $60 \times 84 \mathrm{ps}=5.04 \mathrm{~ns}$ (Fig. 20) with a bandwidth of $1 / \Delta t=11.9$ GHz. Figs. 20(a)-(c) show the results with different Gaussian pulse RF signals with a width varying from $0.20 \mathrm{~ns}$ to $0.94 \mathrm{~ns}$, and a time window $T(\sim 5 \mathrm{~ns})$ that agrees with theory $(5.04 \mathrm{~ns})$. Figures $20(\mathrm{~d})-(\mathrm{e})$ show the integration of dual Gaussian pulses separated by $1.52 \mathrm{~ns}$ and $3.06 \mathrm{~ns}$, where the integrator displays three distinct intensity steps. The left step corresponds to integration of the first pulse while the middle step is the integration of both pulses and the right step is the integration of only the second pulse, since that pulse lies outside the time window of the first pulse. The integrator was then tested with a rectangular input waveform with a width equal to the integration window (5 ns). The measured integrated waveform exhibited a triangular shape that agreed with theory.

Figure 20 shows small discrepancies between experiment and theory - a result of the non-ideal impulse response of the system arising from small effects such as the wavelength variation of the optical amplifier gain, and the photodetector and modulator responses. To verify this, the system impulse response to a Gaussian pulse input was measured. Since the system time resolution $(\sim 84 \mathrm{ps})$ was much less than the pulse width, the wavelength channels were separated into multiple subsets, each with a much larger spacing between the adjacent comb lines and so obtaining a temporal resolution larger than the input pulse duration, and their impulse response was then sequentially measured. Figure 21(a) shows the measured system impulse response which was not flat even if the comb lines were perfectly uniform. The RF signal and impulse response in Fig. 20 were used to calculate the integral output, with the results matching well with experiment, indicating that errors were induced by the non-ideal system impulse response. These errors are in fact easy to compensate for by, for example, using more accurate comb shaping where the feedback loop error signal is generated directly by the measured impulse response and not the optical power of the comb lines. Hence, this implicitly compensated for the wavelength dependence of all components. A flat impulse response resulted, (Fig. 21(b)), much closer to the ideal response than Fig. 21(a). Integration was then performed for the same RF signals and the results are shown in Fig. 22. During these measurements, 81 wavelength channels were used by the impulse response shaping process, yielding an time integration window $(T=N \times \Delta t)$ that was increased to $81 \times 84 \mathrm{ps}=6.804$ $\mathrm{ns}$, resulting in a bandwidth of $1 / 84 \mathrm{ps}=11.9 \mathrm{GHz}$ and time-bandwidth product of $6.804 \mathrm{~ns} \times 11.9 \mathrm{GHz}=\sim 81$ (approximately equal to $N$, the number of channels). The measured integrals (Fig. 22) show much better agreement with theory, indicating the advantage of the shaping impulse response compensating method as well as the overall power and feasibility of our RF integrator. 


\section{DISCUSSION}

We have reviewed recent progress on the use of Kerr micro-combs for high bandwidth temporal RF and microwave signal processing, focusing on integral and fractional order Hilbert transformers, high order and fractional differentiators, both with continuously tunability in their order, and finally RF integrators. Micro-combs produce a large number of comb lines that greatly increase the performance and processing bandwidth of RF systems in both amplitude and phase response. They have achieved powerful results for RF signal processing because of their large number of comb lines, compact footprint, and large comb spacings compared to other approaches such as electro-optic combs. The devices achieved operation bandwidths of $\sim 16 \mathrm{GHz}$, for the $49 \mathrm{GHz}$ devices and $\sim 25 \mathrm{GHz}$ for $200 \mathrm{GHz}$ FSR combs, evaluated in the frequency domain with Vector Network Analyzers and time domain with Gaussian pulses.

The performance of frequency domain signal processors including Hilbert transformers and differentiators is determined by the number of wavelengths, or taps. We have reviewed the benefit of Kerr micro-combs for photonic $\mathrm{RF}$ and microwave transversal filters, showing the contrast between using widely spaced versus low spaced microcombs - ie., $200 \mathrm{GHz}$ versus $49 \mathrm{GHz}$ combs, in performance. The 80 lines supplied by the $49 \mathrm{GHz}$ comb (vs 20 for the 200-GHz comb) produced much better performance. In contrast, the 49-GHz device has a lower bandwidth, being limited to the $25 \mathrm{GHz}$ Nyquist zone, while the 200-GHz devices had RF bandwidths well beyond standard electronic microwave technology.

Two types of combs employed to realize RF functions were qualitatively very different. Apart from the spacings being very different, the widely spaced $200 \mathrm{GHz}$ FSR microcomb operated in a quasi-coherent state that did not feature solitons, whereas the low spaced microcomb with an FSR of $49 \mathrm{GHz}$ operated in a state referred to as soliton crystals. Within the optical $\mathrm{C}$ band, the $49 \mathrm{GHz}$ spaced microcomb enabled up to $90 \mathrm{comb}$ lines, yielding significantly better performance for the temporal signal processors than the than the $200 \mathrm{GHz}$ spaced microcomb which only allowed 20 taps in the C-band. Further, the soliton crystal states offered many other advantages such as much lower noise, higher stability, and much easier generation including the ability to even initiate soliton crystals through simple manual tuning of the pump wavelength. However, on the other hand the $49 \mathrm{GHz}$ combs generally yielded much smaller Nyquist bandwidths typically near $\sim 16 \mathrm{GHz}$. For the transversal temporal signal processors such as the integrators, the large number of wavelengths brought about a large number of broadcasted RF replicas, thus yielding a large integration window for the integrator. The temporal response of the integrator was measured with a diverse range of RF inputs, verifying a integration window of $6.8 \mathrm{~ns}$ and a time feature as fast as $84 \mathrm{ps}$. We achieve excellent agreement of our experimental results with theory, confirming that our approach is effective for reconfigurable high-speed signal processing with high bandwidths for microwave systems.

Micro-combs offer many benefits for photonic RF signal processing. First, the coherent soliton states will enable advanced RF functions such as clock generation and wideband frequency conversion. Maturing nanofabrication techniques can achieve high Q MRRs for comb generation with FSRs from 10s' of GHz [119] to THz [47], covering the full RF bands of interest for almost any integrated RF system. The wide range in FSRs of micro-combs yields a large range in Nyquist zone to 100's of GHz, well beyond electronics and also challenging even for mode-locked lasers and EO modulation OFCs. Using tailored dispersion, micro-combs with ultra-wideband bandwidths - even octavespanning, [71] can be achieved, enabling a very large number of wavelengths using only a single integrated source, to work in conjunction with broadband opto-electronic equipment. This approach enables significantly enhanced wavelength-division parallelism for massive data transmission and processing [62-64] for many applications including radio-over-fibre systems, for example.

Perhaps most importantly, the high reconfigurability of micro-comb based programmable RF photonic transversal filters enables one to achieve highly versatile processing functions with dynamic real-time reconfigurability so that the same system can be dynamically reprogrammed to perform a wide range of different functions without having to alter any hardware. Simply by programming the comb line intensities according to the required tap weights, the same hardware can achieve many different signal processing functions. This very high level of reconfigurability is unprecedented for photonic RF signal processors and enables highly reconfigurable processing functions and high processing accuracy that typically cannot be obtained by passive photonic integrated circuits or via optical analogue signal processing. Further, very wide operational RF bandwidths can be achieved for diverse computing requirements of many practical applications. Photonic transversal filters are similar to filters achieved using digital electronic signal processing except realized with photonics. 
There are a number of factors that can lead to tap errors during the comb shaping, thus leading to non-ideal impulse responses of the system as well as deviations between the experimental results and theory. These factors include optical micro-comb instability, waveshaper accuracy, wavelength variation in optical amplifier gain, modulator induced chirp, and dispersive fibre third-order dispersion. These errors can be reduce by using feedback control paths in real-time, where the comb line power is detected by an OSA and compared with the theoretical tap weights, generating an error signal fed back to the waveshaper for accurate comb shaping. The best way of generating the error signal is directly from the measured impulse response instead of the raw optical power of the comb lines. In this approach, the impulse response of the system is obtained by measuring replicas of an RF Gaussian pulse at all wavelengths, further extracting the peak intensities to obtain accurate RF-to-RF wavelength channel weights. After this, the measured channel weights are subtracted from the theoretical weights to obtain an error signal then used to set the Waveshaper loss. After several iterations of comb shaping an accurate impulse response that compensates the non-ideal impulse response of the system can be obtained, thus significantly improving the RF photonic transversal filter accuracy. This calibration process only needs to be done once.

Finally, although the results reviewed here relied on some benchtop components, such as the commercially available Waveshaper, RF photonic signal processors based on Kerr micro-combs have significant potential to achieve much higher levels of integration with current nanofabrication techniques. To begin with, the most important component is the micro-comb source itself, which not only is already integrated but is fabricated with CMOS compatible processes. CMOS fabrication foundries can already perform advanced hybrid integration of microcombs with III-V devices, and this will ultimately enable the monolithic integration of entire RF systems. Other key components have been realized in integrated form with state-of-art nanofabrication techniques [198-200], including the optical pump source [131, 139], $\mathrm{LiNO}_{3}$ modulators [200], optical spectral shapers [198], large dispersion media [199] and photodetectors. Further, advanced integrated microcombs have been demonstrated [139] that can generate soliton crystals reliably in turn-key operation. Monolithically integrating the whole RF processing system would greatly strengthen the performance, compactness and energy efficiency of the system. Even without this, however, using the discrete integrated comb sources to replace discrete laser arrays already yields significant benefits for RF and microwave systems in terms of performance, size, cost, and complexity. These platforms have already had an enormous impact on microwave photonics, quantum optics and integration with ultrahigh nonlinearity 2D materials [201-277].

\section{CONCLUSION}

We review progress in applications of Kerr micro-combs to RF and microwave photonic time based signal processing using transversal filters. Optical micro-combs are a new generation of multi-wavelength compact sources for the RF photonics community to exploit, offering huge benefits for high-performance RF signal processing with reduced footprint, complexity, and potentially cost. By programming the comb lines according to calculated tap weights, a wide range of signal processing functions have been experimentally demonstrated. We have focused on fractional and integral order Hilbert transforms and differentiators as well as RF integrators that operate on the RF signal rather than the complex optical field. Real-time system demonstrations are also performed that show good agreement with theory. These results verify that Kerr micro-combs are highly effective in achieving high-speed reconfigurable signal processing for future ultra-high performance RF systems

\section{References}

[1] S. Radic, and D. J. Moss, and B. J. Eggleton, "Nonlinear optics in communications: From crippling impairment to ultrafast tools", Chapter 20, p759-828 in Optical Fiber Telecommunications V: Components and Sub-systems, Ed. Ivan P. Kaminow, Tingye Li, and Alan E. Willner, Academic Press, Oxford, UK, February 2008.

[2] J. Leuthold, C. Koos, and W. Freude, "Nonlinear silicon photonics,” Nat. Photon., vol. 4, no. 8, pp. 535-544, 2010.

[3] L. Li, P. G. Patki, Y.B. Kwon, et al. "All-optical regenerator of multi-channel signals," Nature Communications, vol. 8, Article number: 884, 2017.

[4] F. Li, et al., "All-optical XOR logic gate for 40Gb/s DPSK signals via FWM in a silicon nanowire," Optics Express, vol. 19, no. 21, pp. 20364-20371, 2011. DOI: 10.1364/OE.19.020364. 
[5] F. Li, et al., "Error-free All-Optical Demultiplexing at 160Gb/s via FWM in a Silicon Nanowire," Optics Express, vol. 18, no. 4, pp. 3905-3910, 2010. DOI: 10.1364/OE.18.003905.

[6] H. Ji, et al., "1.28-Tb/s Demultiplexing of an OTDM DPSK Data Signal Using a Silicon Waveguide," Photonics Technology Letters, vol. 22, no. 23, pp. 1762-1764, 2010.

[7] C. Monat, et al., "Investigation of phase matching for third-harmonic generation in silicon slow light photonic crystal waveguides using Fourier optics," Optics Express, vol. 18, no. 7, pp. 6831-6840, 2010. DOI: 10.1364/OE.18.006831.

[8] B. Corcoran, et al., "Optical Signal Processing on a Silicon Chip at 640Gb/s Using Slow-Light," Optics Express, vol. 18, no. 8, pp. 7770-7781, 2010. DOI: 10.1364/OE.18.007770.

[9] V. G. Ta'eed, et al., "Integrated all-optical pulse regenerator in chalcogenide waveguides," Optics Letters, vol. 30, no. 21, pp. 2900-2902, 2005. DOI: 10.1364/OL.30.002900.

[10] M. Rochette, et al., "Bit-error-ratio improvement with 2R optical regenerators," IEEE Photonics Technology Letters, vol. 17, no. 4, pp. 908-910, 2005.

[11] M. Ferrera, et al., "CMOS compatible integrated all-optical radio frequency spectrum analyzer," Optics Express, vol. 22, no. 18, pp. 21488 - 21498, 2014. DOI: 10.1364/OE.22.021488.

[12] C. Monat, et al., "Integrated optical auto-correlator based on third-harmonic generation in a silicon photonic crystal waveguide," Nature Communications, vol. 5, Article:3246, 2014. doi:10.1038/ncomms4246.

[13] F. Li, et al., "All-optical wavelength conversion for $10 \mathrm{~Gb} / \mathrm{s}$ DPSK signals in a silicon ring resonator," Optics Express, vol. 19, no. 23, pp. 22410-22416, 2011.

[14] T. D. Vo, et al., "Silicon-Chip-Based Real-Time Dispersion Monitoring for 640 Gbit/s DPSK Signals," IEEE Journal of Lightwave Technology, vol. 29, no. 12, pp. 1790-1796, 2011.

[15] M. Ferrera, et al., "All-optical $1^{\text {st }}$ and $2^{\text {nd }}$ order integration on a chip,” Optics Express, vol. 19, no. 23, pp. 23153-23161, 2011.

[16] B. Corcoran, et al., "Silicon nanowire based radio-frequency spectrum analyzer," Optics Express, vol. 18, no. 19, pp. 2019020200, 2010. DOI: 10.1364/OE.18.020190.

[17] B. Corcoran, et al., "Green light emission in silicon through slow-light enhanced third-harmonic generation in photonic-crystal waveguides," Nature Photonics, vol. 3, no. 4, pp. 206-210, 2009. doi:10.1038/nphoton.2009.28.

[18] D. J. Moss, H. M. van Driel, and J. E. Sipe, "Dispersion in the anisotropy of optical third-harmonic generation in silicon," Opt. Lett., vol. 14, no. 1, pp. 57-59, 1989.

[19] J. E. Sipe, D. J. Moss, and H. M. van Driel, "Phenomenological Theory of Optical Second- And Third-Harmonic Generation Form Cubic Centrosymmetric Crystals," Phys. Rev. B, vol. 35, no. 3, pp. 1129-1141, 1987.

[20] D. J. Moss, E. Ghahramani, J. E. Sipe, and H. M. van Driel, "Band-structure calculation of dispersion and anisotropy in $\chi \rightarrow(3)$ for third-harmonic generation in Si, Ge, and GaAs," Phys. Rev. B, vol. 41, no. 3, pp. 1542-1560, 1990.

[21] D. J. Moss, H. M. van Driel, and J. E. Sipe, "Third harmonic generation as a structural diagnostic of ion implanted amorphous and crystalline silicon," Appl. Phy. Lett., vol. 48, no. 17, pp. 1150, 1986.

[22] D. J. Moss, et al., "Ultrafast all-optical modulation via two-photon absorption in silicon-insulator waveguides," Electronics Letters, vol. 41, no. 6, pp. 320-321, 2005. DOI:10.1049/el:20058051

[23] M. R. E. Lamont, et al.,"Two-photon absorption effects on self-phase-modulation-based 2R optical regeneration," Photonics Technology Letters, vol. 18, no. 10, pp. 1185-1187, 2006. DOI:10.1109/LPT.2006.874718.

[24] A.Tuniz, G. Brawley, D. J. Moss, and B. J. Eggleton, "Two-photon absorption effects on Raman gain in single mode As2Se3 chalcogenide glass fiber," Optics Express, vol. 16, no. 22, pp. 18524-18534, 2008. DOI: 10.1364/OE.16.018524.

[25] M.D. Pelusi, F. Luan, E. Magi, M.R.E. Lamont, D. J. Moss, B.J. Eggleton, J.S. Sanghera, L. B. Shaw, and I.D. Aggarwal, "High bit rate all-optical signal processing in a fiber photonic wire", Optics Express vol.16, 11506-11512 (2008). 
[26] M. Lee, et al., "Photosensitive post tuning of chalcogenide photonic crystal waveguides," Optics Express, vol. 15, no. 3, pp. 12771285, 2007. DOI:10.1364/OE.15.001277

[27] S. Tomljenovic-Hanic, M. J. Steel, C. M. d. Sterke and D. J. Moss, "High-Q cavities in photosensitive photonic crystals", Optics Letters, 32, no. 5, pp. 542-544, 2007.

[28] C. Grillet, et al., "Nanowire coupling to photonic crystal nanocavities for single photon sources," Optics Express, vol. 15, no. 3, pp. 1267-1276, 2007. DOI:10.1364/OE.15.001267

[29] V. G. Ta'eed, et al., "Ultrafast all-optical chalcogenide glass photonic circuits", Optics Express, vol. 15, no. 15, pp. 9205-9221, 2007.

[30] D. Freeman, et al., "Chalcogenide Glass Photonic Crystal Devices", Photonic and Electromagnetic Crystal Structures, Photonics and Nanostructures-Fundamentals and Applications, Science Direct Elsevier Publishing, vol. 6, no. 1, pp. 3-11, 2008. doi:10.1016/j.photonics.2007.11.001.

[31] C. Grillet, et al., "Characterization and modeling of Fano resonances in chalcogenide photonic crystal membranes", Optics Express, vol. 14, no. 1, pp. 369-376, 2006.

[32] V. G. Ta'eed, et al., "Self-phase modulation based integrated optical regeneration in chalcogenide waveguides", IEEE Journal of Selected Topics in Quantum Electronics, vol. 12, no. 3, pp. 360-370, 2006.

[33] M. Shokooh-Saremi, et al., "High performance Bragg gratings in chalcogenide rib waveguides written with a modified Sagnac interferometer: experiment and modeling", Journal of the Optical Society of America B (JOSA B), vol. 23, no. 7, pp. 1323-1331, 2006.

[34] M. R. E. Lamont, et al., "Error-free wavelength conversion via cross phase modulation in $5 \mathrm{~cm}$ of As $2 \mathrm{~S} 3$ chalcogenide glass rib waveguide", Electronics Letters, vol. 43, pp. 945-947, 2007.

[35] K. Ikeda, R. E. Saperstein, N. Alic, and Y. Fainman, "Thermal and Kerr nonlinear properties of plasma-deposited silicon nitride/silicon dioxide waveguides”, Opt. Express, vol. 16, pp. 12987-12994, 2008.

[36] J. S. Levy, A. Gondarenko, M. A. Foster, et al., "CMOS-compatible multiple-wavelength oscillator for on-chip optical interconnects," Nature Photonics, vol. 4, 1, pp. 37-40, 2010.

[37] L. Razzari, D. Duchesne, M. Ferrera, et al., "CMOS-compatible integrated optical hyper-parametric oscillator," Nature Photonics, vol. 4, no. 1, pp. 41-45, 2010.

[38] D. J. Moss, R. Morandotti, A. L. Gaeta, et al., "New CMOS-compatible platforms based on silicon nitride and Hydex for nonlinear optics," Nature Photonics, vol. 7, no. 8, pp. 597-607, 2013.

[39] M. Ferrera, L. Razzari, D. Duchesne, et al., "Low-power continuous-wave nonlinear optics in doped silica glass integrated waveguide structures," Nature Photonics, vol. 2, no. 12, pp. 737-740, 2008.

[40] M. Ferrera, D. Duchesne, L. Razzari, et al., "Low power four wave mixing in an integrated, micro-ring resonator with $\mathrm{Q}=1.2$ million," Optics Express, vol. 17, no. 16, pp. 14098-14103, 2009.

[41] D. Duchesne, M. Peccianti, M. R. E. Lamont, et al., "Supercontinuum generation in a high index doped silica glass spiral waveguide," Optics Express, vol. 18, no, 2, pp. 923-930, 2010.

[42] M. Ferrera, et al., "On-chip CMOS-compatible all-optical integrator", Nature Communications, vol. 1, Article $29,2010$. DOI: $10.1038 /$ ncomms 1028

[43] A. Pasquazi, et al., "All-optical wavelength conversion in an integrated ring resonator," Optics Express, vol. 18, no. 4, pp. 38583863, 2010.

[44] A. Pasquazi, Y. Park, J. Azana, et al., "Efficient wavelength conversion and net parametric gain via Four Wave Mixing in a high index doped silica waveguide," Optics Express, vol. 18, no. 8, pp. 7634-7641, 2010.

[45] M. Peccianti, M. Ferrera, L. Razzari, et al., "Subpicosecond optical pulse compression via an integrated nonlinear chirper," Optics Express, vol. 18, no. 8, pp. 7625-7633, 2010.

[46] D. Duchesne, M. Ferrera, L. Razzari, et al., "Efficient self-phase modulation in low loss, high index doped silica glass integrated waveguides," Optics Express, vol. 17, no. 3, pp. 1865-1870, 2009.

[47] A. Pasquazi, M. Peccianti, L. Razzari, D. J. Moss, S. Coen, M. Erkintalo, Y. K. Chembo, T. Hansson, S. Wabnitz, P. Del'Haye, X. X. Xue, A. M. Weiner, and R. Morandotti, "Micro-combs: A novel generation of optical sources," Physics Reports, vol. 729, pp. 1-81, Jan 27. 2018. 
[48] P. Del'Haye, A. Schliesser, O. Arcizet, T. Wilken, R. Holzwarth, and T. J. Kippenberg, "Optical frequency comb generation from a monolithic microresonator", Nature, vol. 450, pp. 1214-1217, 2007.

[49] M. Peccianti, et al., "Demonstration of an ultrafast nonlinear microcavity modelocked laser", Nature Communications, vol. 3, pp. 765, 2012. DOI:10.1038/ncomms 1762

[50] M. Kues, et al., "Passively modelocked laser with an ultra-narrow spectral width", Nature Photonics, vol. 11, no. 3, pp. $159,2017$. DOI:10.1038/nphoton.2016.271

[51] A. Pasquazi, L. Caspani, M. Peccianti, et al., "Self-locked optical parametric oscillation in a CMOS compatible microring resonator: a route to robust optical frequency comb generation on a chip," Optics Express, vol. 21, no. 11, pp. 13333-13341, 2013.

[52] A. Pasquazi, M. Peccianti, B. E. Little, et al., "Stable, dual mode, high repetition rate mode-locked laser based on a microring resonator," Optics Express, vol. 20, no. 24, pp. 27355-27362, 2012.

[53] C. Reimer, L. Caspani, M. Clerici, et al., "Integrated frequency comb source of heralded single photons," Optics Express, vol. 22, no. 6, pp. 6535-6546, 2014.

[54] C. Reimer, et al., "Cross-polarized photon-pair generation and bi-chromatically pumped optical parametric oscillation on a chip", Nature Communications, vol. 6, Article 8236, 2015. DOI: 10.1038/ncomms9236

[55] L. Caspani, C. Reimer, M. Kues, et al., "Multifrequency sources of quantum correlated photon pairs on-chip: a path toward integrated Quantum Frequency Combs," Nanophotonics, vol. 5, no. 2, pp. 351-362, 2016.

[56] C. Reimer, M. Kues, P. Roztocki, B. Wetzel, F. Grazioso, B. E. Little, S. T. Chu, T. Johnston, Y. Bromberg, L. Caspani, D. J. Moss, and R. Morandotti, "Generation of multiphoton entangled quantum states by means of integrated frequency combs," Science, vol. 351, no. 6278, pp. 1176-1180, 2016.

[57] M. Kues, et al., "On-chip generation of high-dimensional entangled quantum states and their coherent control", Nature, vol. 546, no. 7660, pp. 622-626, 2017.

[58] P. Roztocki, M. Kues, C. Reimer, B. Wetzel, S. Sciara, Y. Zhang, A. Cino, B. E. Little, S. T. Chu, D. J. Moss, and R. Morandotti, "Practical system for the generation of pulsed quantum frequency combs," Optics Express, vol. 25, no. 16, pp. 18940-18949, 2017.

[59] Y. Zhang, et al., "Induced photon correlations through superposition of two four-wave mixing processes in integrated cavities", Laser and Photonics Reviews, vol. 14, no. 7, pp. 2000128, 2020. DOI: 10.1002/lpor.202000128

[60] M. Kues, C. Reimer, A. Weiner, J. Lukens, W. Munro, D. J. Moss, and R. Morandotti, "Quantum Optical Micro-combs", Nature Photonics, vol. 13, no.3, pp. 170-179, 2019.

[61] C. Reimer, et al.,"High-dimensional one-way quantum processing implemented on d-level cluster states", Nature Physics, vol. 15, no.2, pp. 148-153, 2019.

[62] P. Marin-Palomo, et al., "Microresonator-based solitons for massively parallel coherent optical communications", Nature, vol. 546, no. 7657, pp. 274, 2017.

[63] J. Pfeifle, V. Brasch, M. Lauermann, Y. Yu, D. Wegner, T. Herr, K. Hartinger, et al., "Coherent terabit communications with microresonator Kerr frequency combs”, Nature Photonics, vol. 8, no. 5, pp. 375-380, 2014.

[64] B. Corcoran, et al., "Ultra-dense optical data transmission over standard fiber with a single chip source", Nature Communications, vol. 11, Article:2568, 2020. DOI:10.1038/s41467-020-16265-x.

[65] X. Xu, et al., "Photonic perceptron based on a Kerr microcomb for scalable high speed optical neural networks", Laser and Photonics Reviews, vol. 14, no. 8, 2000070, 2020. DOI:10.1002/lpor.202000070.

[66] X. Xu, et al., "11 TOPs photonic convolutional accelerator for optical neural networks", Nature, vol.589 (7840) 44-51 (2021). DOI: $10.1038 / \mathrm{s} 41586-020-03063-0$.

[67] J. Feldmann, et al., "Parallel convolution processing using an integrated photonic tensor core", arXiv preprint arXiv:2002.00281, 2020.

[68] D. T. Spencer, et al., "An optical-frequency synthesizer using integrated photonics", Nature, vol. 557, no. 7703, pp. 81-85, 2018.

[69] T. J. Kippenberg, A. L. Gaeta, M. Lipson, and M. L. Gorodetsky, "Dissipative Kerr solitons in optical microresonators," Science, vol. 361, no. 6402, 2018.

[70] A. L. Gaeta, M. Lipson, and T. J. Kippenberg, "Photonic-chip-based frequency combs," Nature Photonics, vol. 13, no. 3, pp. 158169, Mar. 2019.

[71] P. Del'Haye, T. Herr, E. Gavartin, M. L. Gorodetsky, R. Holzwarth, and T. J. Kippenberg, "Octave spanning tunable frequency comb from a microresonator”, Physical Review Letters, vol. 107, no. 6, pp. 063901, 2011. 
[72] T. J. Kippenberg, R. Holzwarth, S. A. Diddams, "Microresonator-based optical frequency combs", Science, vol. 332, no. 6029, pp. 555-559, 2011.

[73] T. Herr, V. Brasch, J. D. Jost, C. Y. Wang, N. M. Kondratiev, M. L. Gorodetsky, et al., "Temporal solitons in optical microresonators", Nature Photonics, vol. 8, no. 2, pp. 145-152, 2014.

[74] F. Ferdous, H. Miao, D. E. Leaird, K. Srinivasan, J. Wang, L. Chen, L. T. Varghese, and A. M. Weiner, "Spectral line-by-line pulse shaping of on-chip microresonator frequency combs", Nature Photonics, vol. 5, no. 12, pp. 770, 2011.

[75] X. Xue, P. H. Wang, Y. Xuan, M. Qi, and A. M. Weiner, "Microresonator Kerr frequency combs with high conversion efficiency", Laser \& Photonics Reviews, vol. 11, no. 1, 2017.

[76] X. Xue, M. Qi, and A. M. Weiner, "Normal-dispersion microresonator Kerr frequency combs", Nanophotonics, vol. 5, no. 2, pp. 244-262, 2016.

[77] C. Grillet, et al., "Amorphous Silicon Nanowires with Record High Nonlinearity, FOM, and Optical Stability", Optics Express, vol. 20, no. 20. pp. 22609-22615, 2012.

[78] J. W. Choi, B. Sohn, G. F. R. Chen, D. K. T. Ng, and D. T. H. Tan, "Soliton-effect optical pulse compression in CMOS-compatible ultra-silicon-rich nitride waveguides," APL Photonics, HIBSP2019, pp. 110804, 2020.

[79] J. Capmany, and D. Novak, "Microwave photonics combines two worlds," Nat. Photonics, vol. 1, no. 6, pp. 319-330, 2007.

[80] J. P. Yao, “Microwave photonics," Journal of Lightwave Technol., vol. 27, no. 1-4, pp. 314-335, Jan-Feb. 2009.

[81] D. Marpaung, J. Yao, and J. Capmany, "Integrated microwave photonics," Nature Photonics, vol. 13, no. 2, pp. 80-90, Feb. 2019.

[82] J. Azaña, "Ultrafast analog all-optical signal processors based on fiber-grating devices," IEEE Photonics Journal, vol. 2, no. 3, pp. 359-386. 2010.

[83] J. Capmany, B. Ortega, and D. Pastor, "A tutorial on microwave photonic filters," Journal of Lightwave Technol., vol. 24, no. 1, pp. 201-229, 2006.

[84] V. R. Supradeepa et al., "Comb-based radiofrequency photonic filters with rapid tunability and high selectivity," Nat. Photonics, vol. 6, no. 3, pp. 186-194, Mar.2012.

[85] J. Wu, X. Xu, T. G. Nguyen, S. T. Chu, B. E. Little, R. Morandotti, A. Mitchell, and D. J. Moss, "RF Photonics: An Optical Microcombs' Perspective," IEEE J. Sel. Top. Quantum Electron., vol. 24, no. 4, pp. 6101020, Jul-Aug. 2018. DOI: 10.1109/JSTQE.2018.2805814.

[86] V. Torres-Company, and A. M. Weiner, "Optical frequency comb technology for ultra-broadband radio-frequency photonics," Laser Photonics Rev, vol. 8, no. 3, pp. 368-393, May. 2014.

[87] Z. Jiang, C. B. Huang, D. E. Leaird, and A. M. Weiner, "Optical arbitrary waveform processing of more than 100 spectral comb lines," Nat Photonics, vol. 1, no. 8, pp. 463-467, Aug. 2007.

[88] Y. Liu, J. Hotten, A. Choudhary, B. J. Eggleton, and D. Marpaung, "All-optimized integrated RF photonic notch filter," Opt. Lett., vol. 42, no. 22, pp. 4631-4634, Nov 15. 2017.

[89] Y. Liu, D. Marpaung, A. Choudhary, J. Hotten, and B. J. Eggleton, "Link performance optimization of chip-based Si3N4 microwave photonic filters," J. Lightwave Technol., vol. 36, no. 19, pp. 4361-4370, 2018.

[90] Y. Liu, Y. Yu, S. X. Yuan, X. B. Xu, and X. L. Zhang, "Tunable megahertz bandwidth microwave photonic notch filter based on a silica microsphere cavity," Opt. Lett., vol. 41, no. 21, pp. 5078-5081, Nov 1. 2016.

[91] D. Marpaung, B. Morrison, M. Pagani, R. Pant, D. Y. Choi, B. Luther-Davies, S. J. Madden, and B. J. Eggleton, "Low-power, chip-based stimulated Brillouin scattering microwave photonic filter with ultrahigh selectivity," Optica, vol. 2, no. 2, pp. 76-83, Feb 20. 2015.

[92] A. Choudhary, B. Morrison, I. Aryanfar, S. Shahnia, M. Pagani, Y. Liu, K. Vu, S. Madden, D. Marpaung, and B. J. Eggleton, "Advanced integrated microwave signal processing with giant on-chip Brillouin gain," J. Lightwave Technol., vol. 35, no. 4, pp. 846-854, Feb 15. 2017.

[93] D. Marpaung, B. Morrison, R. Pant, and B. J. Eggleton, "Frequency agile microwave photonic notch filter with anomalously high stopband rejection," Opt. Lett., vol. 38, no. 21, pp. 4300-4303, Nov 1. 2013.

[94] X. Q. Zhu, F. Y. Chen, H. F. Peng, and Z. Y. Chen, "Novel programmable microwave photonic filter with arbitrary filtering shape and linear phase," Opt. Express, vol. 25, no. 8, pp. 9232-9243, Apr 17. 2017.

[95] F. Jiang, Y. Yu, H. T. Tang, L. Xu, and X. L. Zhang, "Tunable bandpass microwave photonic filter with ultrahigh stopband attenuation and skirt selectivity," Opt. Express, vol. 24, no. 16, pp. 18655-18663, Aug 8. 2016. 
[96] Z. J. Zhu, H. Chi, T. Jin, S. L. Zheng, X. F. Jin, and X. M. Zhang, "All-positive-coefficient microwave photonic filter with rectangular response," Opt. Lett., vol. 42, no. 15, pp. 3012-3015, Aug 1. 2017.

[97] G. Yu, W. Zhang, and J. A. R. Williams, "High-performance microwave transversal filter using fiber Bragg grating arrays," IEEE Photonic Tech L, vol. 12, no. 9, pp. 1183-1185, Sep. 2000.

[98] J. S. Leng, W. Zhang, and J. A. R. Williams, "Optimization of superstructured fiber Bragg gratings for microwave photonic filters response,” IEEE Photonic Tech L, vol. 16, no. 7, pp. 1736-1738, Jul. 2004.

[99] D. B. Hunter, R. A. Minasian, and P. A. Krug, "Tunable optical transversal filter based on chirped gratings," Electron. Lett., vol. 31, no. 25, pp.2205-2207, Dec 7. 1995.

[100] E. Hamidi, D. E. Leaird, and A. M. Weiner, "Tunable Programmable Microwave Photonic Filters Based on an Optical Frequency Comb," IEEE Journal of Microwave Theory, vol. 58, no. 11, pp. 3269-3278, Nov. 2010.

[101] R. Wu, V. R. Supradeepa, C. M. Long, D. E. Leaird, and A. M. Weiner, "Generation of very flat optical frequency combs from continuous-wave lasers using cascaded intensity and phase modulators driven by tailored radio frequency waveforms," Opt. Lett., vol. 35, no. 19, pp. 3234-3236, Oct 1. 2010.

[102] S. Mansoori, and A. Mitchell, "RF transversal filter using an AOTF," IEEE Photonic Tech L, vol. 16, no. 3, pp. 879-881, Mar. 2004.

[103] M. Delgado-Pinar, J. Mora, A. Diez, M. V. Andres, B. Ortega, and J. Capmany, "Tunable and reconfigurable microwave filter by use of a Bragg-grating-based acousto-optic superlattice modulator," Opt. Lett., vol. 30, no. 1, pp. 8-10, Jan 1. 2005.

[104] W. Z. Li, and J. P. Yao, "Optical frequency comb generation based on repeated frequency shifting using two Mach-Zehnder modulators and an asymmetric Mach-Zehnder interferometer," Opt. Express, vol. 17, no. 26, pp. 23712-23718, Dec 21. 2009.

[105] C. H. Chen, C. He, D. Zhu, R. H. Guo, F. Z. Zhang, and S. L. Pan, "Generation of a flat optical frequency comb based on a cascaded polarization modulator and phase modulator," Opt. Lett., vol. 38, no. 16, pp. 3137-3139, Aug 15. 2013.

[106] T. Saitoh, M. Kourogi, and M. Ohtsu, "An optical frequency synthesizer using a waveguide-type optical frequency comb generator at 1.5-mu m wavelength," IEEE Photonic Tech L, vol. 8, no. 11, pp. 1543-1545, Nov. 1996.

[107] T. G. Nguyen et al., "Integrated frequency comb source-based Hilbert transformer for wideband microwave photonic phase analysis," Opt. Express, vol. 23, no. 17, pp. 22087-22097, Aug. 2015.

[108] X. Xue, et al., "Programmable single-bandpass photonic RF filter based on a Kerr comb from a microring," Journal of Lightwave Technol., vol. 32, no. 20, pp. 3557-3565, Oct. 2014.

[109] X. Xu, J. Wu, M. Shoeiby, T. G. Nguyen, S. T. Chu, B. E. Little, R. Morandotti, A. Mitchell, and D. J. Moss, "Reconfigurable broadband microwave photonic intensity differentiator based on an integrated optical frequency comb source," APL Photonics, vol. 2, no. 9, 096104, Sep. 2017.

[110] X. Xu, M. Tan, J. Wu, R. Morandotti, A. Mitchell, and D. J. Moss, "Microcomb-based photonic RF signal processing", IEEE Photonics Technology Letters, vol. 31 no. 23 1854-1857, 2019.

[111] X. Xu, et al., "Advanced RF and microwave functions based on an integrated optical frequency comb source," Opt. Express, vol. 26, no. 3, pp. 2569-2583, Feb. 2018.

[112] X. Xue, et al., "Microcomb-based true-time-delay network for microwave beamforming with arbitrary beam pattern control," Journal of Lightwave Technology, vol. 36, no. 12, pp. 2312-2321, Jun. 2018.

[113] X. Xu, et al., "Broadband RF channelizer based on an integrated optical frequency Kerr comb source," Journal of Lightwave Technology, vol. 36, no. 19, pp. 4519-4526, 2018.

[114] X. Xu, et al., "Continuously tunable orthogonally polarized RF optical single sideband generator based on micro-ring resonators," Journal of Optics, vol. 20, no. 11, 115701. 2018.

[115] X. Xu, et al., "Orthogonally polarized RF optical single sideband generation and dual-channel equalization based on an integrated microring resonator," Journal of Lightwave Technology, vol. 36, no. 20, pp. 4808-4818. 2018.

[116] X. Xu, et al., "Photonic microwave true time delays for phased array antennas using a $49 \mathrm{GHz}$ FSR integrated optical micro-comb source," Photonics Res, vol. 6, no. 5, pp. B30-B36, 2018.

[117] X. Xu, et al., "Advanced adaptive photonic RF filters with 80 taps based on an integrated optical micro-comb source," Journal of Lightwave Technology, vol. 37, no. 4, pp. 1288-1295, 2019.

[118] W. Liang, et al., "High spectral purity Kerr frequency comb radio frequency photonic oscillator," Nature Communications, vol. 6 pp. 7957. 2015. 
[119] J. Liu, et al., "Photonic microwave generation in the X-and K-band using integrated soliton microcombs" Nature Photonics, vol. 14, pp. 1-6, 2020.

[120] X. Xu, et al., Broadband microwave frequency conversion based on an integrated optical micro-comb source", Journal of Lightwave Technology, vol. 38 no. 2, pp. 332-338, 2020.

[121] M. Tan, et al., "Photonic RF and microwave filters based on 49GHz and 200GHz Kerr microcombs", Optics Comm. vol. 465, Article: 125563, Feb. 22. 2020.

[122] X. Xu, et al., "Broadband photonic RF channelizer with 90 channels based on a soliton crystal microcomb", Journal of Lightwave Technology, Vol. 38, no. 18, pp.5116 - 5121, 2020. doi: 10.1109/JLT.2020.2997699.

[123] X. Xu, et al., "Photonic RF and microwave integrator with soliton crystal microcombs", IEEE Transactions on Circuits and Systems II: Express Briefs, Vol.67, Issue: 12, pp. 3582-3586, (2020). DOI:10.1109/TCSII.2020.2995682.

[124] X. Xu, et al., "Photonic RF phase-encoded signal generation with a microcomb source", Journal of Lightwave Technology, vol. 38, no. 7, pp. 1722-1727, 2020.

[125] X. Xu, et al., "High performance RF filters via bandwidth scaling with Kerr micro-combs," APL Photonics, vol. 4, no. 2, pp. 026102. 2019.

[126] M. Tan, et al., "Microwave and RF photonic fractional Hilbert transformer based on a $50 \mathrm{GHz}$ Kerr micro-comb", Journal of Lightwave Technology, vol. 37, no. 24, pp. $6097-6104,2019$.

[127] M. Tan, et al., "RF and microwave fractional differentiator based on photonics", IEEE Transactions on Circuits and Systems: Express Briefs, Vol. 67, Issue: 11, pp.2767-2771 (2020). DOI:10.1109/TCSII.2020.2965158.

[128] M. Tan, et al., "Photonic RF arbitrary waveform generator based on a soliton crystal micro-comb source", Journal of Lightwave Technology, Early Access, vol. 38, (22) Page(s): 6221-6226, Oct 22 (2020). DOI: 10.1109/JLT.2020.3009655.

[129] D. C. Cole, E. S. Lamb, P. Del'Haye, S. A. Diddams, and S. B. Papp, "Soliton crystals in Kerr resonators," Nat. Photonics, vol. 11, no. 10, pp. 671-676, Oct. 2017.

[130] W. Wang, Z. Lu, W. Zhang, S. T. Chu, B. E. Little, L. Wang, X. Xie, M. Liu, Q. Yang, L. Wang, J. Zhao, G. Wang, Q. Sun, Y. Liu, Y. Wang, and W. Zhao, "Robust soliton crystals in a thermally controlled microresonator," Opt. Lett., vol. 43, no. 9, pp. 20022005, 2018.

[131] B. Stern, X. Ji, Y. Okawachi, A. L. Gaeta, and M. Lipson, "Battery-operated integrated frequency comb generator", Nature, vol. 562, no. 7727, pp. 401, 2018.

[132] X. Xue, et al., "Mode-locked dark pulse Kerr combs in normal-dispersion microresonators," Nature Photonics, vol. 9, no. 9, pp. 594. 2015.

[133] H. Bao, et al., "Laser cavity-soliton microcombs," Nature Photonics, vol. 13, no. 6, pp. 384-389, Jun. 2019.

[134] X. Xue, X. Zheng, and B. Zhou, "Super-efficient temporal solitons in mutually coupled optical cavities," Nature Photonics, May 2019.

[135] H. Zhou, et al., "Soliton bursts and deterministic dissipative Kerr soliton generation in auxiliary-assisted microcavities," Light: Science \& Applications, vol. 8, no. 1, pp. 50, 2019.

[136] H. Bao, L. Olivieri, M. Rowley, S. T. Chu, B. E. Little, R. Morandotti, D. J. Moss, J. S. T. Gongora, M. Peccianti and A. Pasquazi, "Turing patterns in a fibre laser with a nested micro-resonator: robust and controllable micro-comb generation", Physical Review Research, vol. 2, pp. 023395, 2020.

[137] L. D. Lauro, J. Li, D. J. Moss, R. Morandotti, S. T. Chu, M. Peccianti, and A. Pasquazi, "Parametric control of thermal selfpulsation in micro-cavities," Opt. Lett. vol. 42, no. 17, pp. 3407-3410, Aug. 2017.

[138] H. Bao, et al., "Type-II micro-comb generation in a filter-driven four wave mixing laser," Photonics Research, vol. 6, no. 5, pp. B67-B73, May 2018.

[139] B. Shen, Chang, L., Liu, J., et al., "Integrated turnkey soliton microcombs," Nature, vol. 582, pp. 365-369, 2020.

[140] D. J. Moss, M. Lamont, S. Mclaughlin, G. Randall, P. Colbourne, S. Kiran and C. A. Hulse,'Tunable dispersion and dispersion slope compensators for 10Gb/s using all-pass multicavity etalons", IEEE Photonics Technology Letters, vol. 15, no. 5, 730-732 (2003). DOI: 10.1109/LPT.2003.809921.

[141] L.M. Lunardi, D.Moss, S.Chandrasekhar, L.L.Buhl, A. Hulse, P.Colbourne, G.Randall, S.Mclaughlin, "Tunable dispersion compensators based on multi-cavity all-pass etalons for 40Gb/s systems", Journal of Lightwave Technology, vol. 20, no. 12, 2136 (2002). DOI: 10.1109/JLT.2002.806768. 
[142] D. J. Moss, et al., "Multichannel tunable dispersion compensation using all-pass multicavity etalons", paper TuT2 Optical Fiber Communications Conference, Anaheim (2002). Post-conference Technical Digest (IEEE Cat. No.02CH37339). Opt Soc. America. Part vol.1, 2002, pp. 132-3. Washington, DC, USA.

[143] L.M. Lunardi, D. Moss, S. Chandrasekhar, L.L. Buhl, “An etalon based tunable dispersion compensator (TDC) device for 40Gb/s applications", European Conference on Optical Communications (ECOC), paper 5.4.6, Copenhagen, Sept. (2002). IEEE. Part vol. 2, 2002, pp. 2 vol. 2. Piscataway, NJ, USA. INSPEC Accession Number: 9153476, Print ISBN: 87-90974-63-8.

[144] S. L.Hahn, Transforms and Applications Handbook, A. D. Poularikas, Ed., 3rd ed. Boca Raton, FL: CRC Press, 2010 , ch. 7.

[145] L. Moura, "Radio Frequency Implementation of the Fractional Hilbert Transform with Transversal Filters," Circuits, Systems \& Signal Processing., vol. 26, no. 3, pp. 407-417, Jun. 2007.

[146] A. W. Lohmann, D. Mendlovic, and Z. Zalevsky, "Fractional Hilbert transform," Opt. Lett., vol. 21, no. 4, pp. 281-283, Feb. 1996.

[147] J. A. Davis, D. E. McNamara, and D. M. Cottrell, “Analysis of the fractional Hilbert transform,” Appl. Opt., vol. 37, no. 29, pp. 6911-6913, Oct. 1998.

[148] C. D. Holdenried, J. W. Haslett, and B. Davies, "A fully integrated 10-Gb/s tapped delay Hilbert transformer for optical single sideband," IEEE Microw. Wireless Compon. Lett., vol. 15, no. 5, pp. 303-305, May 2005.

[149] H. Emami, N. Sarkhosh, L. A. Bui, and A. Mitchell, "Wideband RF photonic in-phase and quadrature-phase generation," Opt. Lett., vol. 33, no. 2, pp. 98-100, Jan 15. 2008.

[150] M. Li, and J. P. Yao, "All-fiber temporal photonic fractional Hilbert transformer based on a directly designed fiber Bragg grating," Opt. Lett., vol. 35, no. 2, pp. 223-225, Jan. 2010.

[151] M. Li, and J. P. Yao, "Experimental Demonstration of a Wideband Photonic Temporal Hilbert Transformer Based on a Single Fiber Bragg Grating," IEEE Photon. Technol. Lett., vol. 22, no. 21 pp. 1559-1561, Nov. 2010.

[152] M. H. Asghari, and J. Azana, "All-optical Hilbert transformer based on a single phase-shifted fiber Bragg grating: design and analysis," Opt. Lett., vol. 34, no. 3, pp. 334-336, Feb. 2009.

[153] T. Yang, J. Dong, L. Liu, S. Liao, S. Tan, L. Shi, D. Gao, and X. Zhang, "Experimental observation of optical differentiation and optical Hilbert transformation using a single SOI microdisk chip," Sci. Rep., vol. 4, pp. 3960, 2014.

[154] W. Liu, et al., "A Fully Reconfigurable Photonic Integrated Signal Processor," Nature Photonics, vol. 10, no. 3, pp. 190-196, 2016.

[155] Z. Zhang, et al., "Wideband and continuously-tunable fractional photonic Hilbert transformer based on a single high-birefringence planar Bragg grating," Optics Express, vol. 26, pp. 20450-20458, 2018.

[156] F. Zeng, and J. Yao, “An Approach to Ultrawideband Pulse Generation and Distribution Over Optical Fiber," IEEE Photonics Technol. Lett., vol. 18, no. 7, pp. 823-825, Apr. 2006.

[157] S. Pan, and J. Yao, "Optical generation of polarity- and shape-switchable ultrawideband pulses using a chirped intensity modulator and a first-order asymmetric Mach-Zehnder interferometer," Opt. Lett., vol. 34, no. 9, pp. 1312-1314, May. 2009.

[158] Y. Yu, J. Dong, X. Li, and X. Zhang, "Ultra-Wideband Generation Based on Cascaded Mach-Zehnder Modulators," IEEE Photonics Technol. Lett., vol. 23, no. 23, Dec. 2011.

[159] L. Zhuang, M. R. Khan, W. Beeker, A. Leinse, R. Heideman, and C. Roeloffzen, "Novel microwave photonic fractional Hilbert transformer using a ring resonator-based optical all-pass filter," Opt. Exp., vol. 20, no. 24, pp. 26499-26510, Nov. 2012.

[160] C. Sima, J. C. Gates, C. Holmes, P. L. Mennea, M. N. Zervas, and P. G. R. Smith, "Terahertz bandwidth photonic Hilbert transformers based on synthesized planar Bragg grating fabrication,” Opt. Lett., vol. 38, no. 17, pp. 3448-3451, Sep. 2013.

[161] H. Shahoei, P. Dumais, and J. P. Yao, "Continuously tunable photonic fractional Hilbert transformer using a high-contrast germanium-doped silica-on-silicon microring resonator," Opt. Lett., vol. 39, no. 9, pp. 2778-2781, May 2014.

[162] Z. Li, Y. Han, H. Chi, X. Zhang, and J. P. Yao, "A continuously Tunable Microwave Fractional Hilbert Transformer Based on a Nonuniformly Spaced Photonic Microwave Delay-Line Filter,” J. Lightwave Technol., vol. 30, no. 12, pp. 1948-1953, Jun. 2012.

[163] Z. Li, H. Chi, X. Zhang, and J. P. Yao, “A Continuously Tunable Microwave Fractional Hilbert Transformer Based on a Photonic Microwave Delay-Line Filter Using a Polarization Modulator," IEEE Photon. Technol. Lett., vol. 23, no. 22, pp. 1694-1696, Nov. 2011.

[164] A. O.-Blanch, J. Mora, J. Capmany, B. Ortega, and D. Pastor, "Tunable radio-frequency photonic filter based on an actively modelocked fiber laser," Opt. Lett., vol. 31, no. 6, pp. 709-711, Mar. 2006.

[165] V. R. Supradeepa, C. M. Long, R. Wu. F. Ferdous, E. Hamidi, D. E. Leaird, and A. M. Weiner, "Comb-based radiofrequency photonic filters with rapid tunability and high selectivity," Nature Photonics, vol. 6, pp. 186-194, Mar. 2012. 
[166] V. T.-Company, and A. M. Weiner, "Optical frequency comb technology for ultra-broadband radio-frequency photonics," Laser Photonics Rev., vol. 8, no. 3, pp. 368-393, 2014.

[167] F. Li, Y. Park, and J. Azaña, "Linear Characterization of Optical Pulses With Durations Ranging From the Picosecond to the Nanosecond Regime Using Ultrafast Photonic Differentiation,” J. Lightw. Technol., vol. 27, no. 1, pp. 4623-4633, 2009.

[168] S. Pan, J. Yao, "Optical generation of polarity- and shape-switchable ultrawideband pulses using a chirped intensity modulator and a first-order asymmetric Mach-Zehnder interferometer," Opt. Lett., vol. 34, no. 9, pp. 1312-1314, 2009.

[169] X. Li, J. Dong, Y. Yu, and X. Zhang, "A Tunable Microwave Photonic Filter Based on an All-Optical Differentiator," IEEE Photon. Technol. Lett., vol. 23, no. 22, pp. 308-310, Mar. 2011.

[170] Y. Han, Z. Li, and J. Yao, "A Microwave Bandpass Differentiator Implemented Based on a Nonuniformly-Spaced Photonic Microwave Delay-Line Filter," J. Lightw. Technol., vol. 29, no. 22, pp. 3470-3475, Nov. 2011.

[171] R. Ashrafi and J. Azaña, "Figure of merit for photonic differentiators," Opt. Exp., vol. 20, no. 3, pp. 2626-2639, Jan. 2012.

[172] F. Zeng and J. Yao, "Ultrawideband Impulse Radio Signal Generation Using a High-Speed Electrooptic Phase Modulator and a Fiber-Bragg-Grating-Based Frequency Discriminator," IEEE Photon. Technol. Lett., vol. 18, no. 19, pp. 2062-2064, Oct. 2006.

[173] P. Li, H. Chen, M. Chen, and S. Xie, "Gigabit/s Photonic Generation, Modulation, and Transmission for a Reconfigurable Impulse Radio UWB Over Fiber System," IEEE Photon. Technol. Lett., vol. 4, no. 3, pp. 805-816, Jun. 2012.

[174] Y. Yu, F. Jiang, H. Tang, L. Xu, X. Liu, J. Dong, and X. Zhang, "Reconfigurable photonic temporal differentiator based on a dualdrive Mach-Zehnder modulator," Opt. Exp., vol. 24, no. 11, pp. 11739-11748, May 2016.

[175] P. Velanas, A. Bogris, A. Argyris, and D. Syvridis, "High-Speed All-Optical First- and Second-Order Differentiators Based on Cross-Phase Modulation in Fibers," J. Lightw. Technol., vol. 26, no. 18, pp. 3269-3276, Sep. 2008.

[176] J. Xu, X. Zhang, J. Dong, D. Liu, and D. Huang, "All-optical differentiator based on cross-gain modulation in semiconductor optical amplifier," Opt. Lett., vol. 32, no. 20, pp. 3029-3031, Oct. 2007.

[177] J. Xu, X. Zhang, J. Dong, D. Liu, and D. Huang, "High-speed all-optical differentiator based on a semiconductor optical amplifier and an optical filter," Opt. Lett., vol. 32, no. 13, pp. 1872-1874, Jul. 2007.

[178] F. Wang, J. Dong, E. Xu, and X. Zhang, "All-optical UWB generation and modulation using SOA-XPM effect and DWDM-based multi-channel frequency discrimination," Opt. Exp., vol. 18, no. 24, pp. 24588-24594, Nov. 2010.

[179] V. Moreno, M. Rius, J. Mora, M. A. Muriel, and J. Capmany, "Integrable high order UWB pulse photonic generator based on cross phase modulation in a SOA-MZI," Opt. Exp., vol. 21, no. 19, pp. 22911-22917, Sep. 2013.

[180] Q. Wang and J. Yao, "Switchable optical UWB monocycle and doublet generation using a reconfigurable photonic microwave delay-line filter," Opt. Exp., vol. 15, no. 22, pp. 14667-14672, Oct. 2007.

[181] M. Bolea, J. Mora, B. Ortega, and J. Capmany, "Optical UWB pulse generator using an $N$ tap microwave photonic filter and phase inversion adaptable to different pulse modulation formats," Opt. Exp., vol. 17, no. 7, pp. 5023-50332, Mar. 2009.

[182] B. Mathieu, P. Melchior, A. Oustaloup, C. Ceyral, "Fractional differentiation for edge detection," Signal Processing, vol. 83, pp. 2421-2432, Nov. 2003.

[183] A. Oustaloup, F. Levron, B. Mathieu, and F. M. Nanot, "Frequency-Band Complex Noninteger Differentiator: Characterization and Synthesis," IEEE Trans. on Circuit and Systems - I: Fundamental Theory and Application, vol. 47, no. 1, pp. 25-39, Jan. 2000.

[184] M.-G. Suh, Q. Yang, K. Yang, X. Yi, and K. J. Vahala, "Microresonator soliton dual-comb spectroscopy," Science, vol. 354, pp. 600-603, Nov. 2016.

[185] R. Slavik, Y. Park, M. Kulishov, R. Morandotti, and J. Azaña, “Ultrafast all-optical differentiator,” Opt. Exp., vol. 14 no, 22 , pp. 10699, 2006.

[186] F. Liu, T. Wang, L. Qiang, T. Ye, Z. Zhang, M. Qiu, and Y. Su, "Compact optical temporal differentiator based on silicon microring," Opt. Exp., vol. 16 no. 20, pp. 15880, 2008.

[187] L. Zhang, J. Wu, X. Yin, X. Sun, P. Cao, X. Jiang, and Y. Su, “A High-Speed Second-Order Photonic Differentiator Based on Two-Stage Silicon Self-Coupled Optical Waveguide,” IEEE Photon. J., vol. 6, no. 2, pp.7900505, 2014.

[188] J. McClellan, T. W. Parks, and L. Rabiner, "A computer program for designing optimum FIR linear phase digital filters," Transactions on Audio and Electroacoustics, vol. 21, no. 6, pp. 506-526, Dec. 1973.

[189] R. C. Williamson, R. D. Esman, “RF Photonics,” Journal of Lightwave Technology, vol. 26, no. 9, pp. 1145-1153, May 2008. 
[190] Y. Park, T.-J. Ahn, Y. Dai, J. Yao, and J. Azaña, “All-optical temporal integration of ultrafast pulse waveforms,” Optics Express, vol. 16, no. 22, pp. 17817-17825, 2008.

[191] R. Slavík, Y. Park, N. Ayotte, S. Doucet, T.-J. Ahn, S. LaRochelle, and J. Azaña, "Photonic temporal integrator for all-optical computing," Optics Express, vol. 16, no. 22, pp. 18202-18214, 2008.

[192] M. H. Asghari, Y. Park, and J. Azaña, "New design for photonic temporal integration with combined high processing speed and long operation time window," Optics Express, vol. 19, no. 2, pp. 425-435, 2011.

[193] W. Liu, M. Li, R. S. Guzzon, E. J. Norberg, J. S. Parker, L. A. Coldren, and J. Yao, "A Photonic Temporal Integrator with an Ultra-Long Integration Time Window based on an InP-InGaAsP Integrated Ring Resonator," Journal of Lightwave Technology, vol. 32, no. 20, pp. 3654-3659, 2014.

[194] Y. Park, and J. Azaña, "Ultrafast photonic intensity integrator," Optics Letters, vol. 34, no. 8, pp. 1156-1158, 2009.

[195] A. Malacarne, R. Ashrafi, M. Li, S. LaRochelle, J. Yao, and J. Azaña, "Single-shot photonic time-intensity integration based on a time-spectrum convolution system," Optics Letters, vol. 37, no. 8, pp. 1355-1357, 2012.

[196] J. Zhang, and J. Yao, "Microwave photonic integrator based on a multichannel fiber Bragg grating," Optics Letters, vol. 41, no. 2, pp. 273-276, 2016.

[197] M.-G. Suh, and K. J. Vahala, “Soliton microcomb range measurement," Science, vol. 359, no. 6378, pp. 884-887, 2018.

[198] A. J. Metcalf, et al., "Integrated line-by-line optical pulse shaper for high-fidelity and rapidly reconfigurable RF-filtering," Optics Express, vol. 24, no. 21, pp. 23925-23940, 2016.

[199] E. Sahin, K. J. A. Ooi, C. E. Png, and D. T. H. Tan, "Large, scalable dispersion engineering using cladding-modulated Bragg gratings on a silicon chip," Applied Physics Letters, vol. 110, pp. 161113, 2017.

[200] C. Wang, et al., "Integrated lithium niobate electro-optic modulators operating at CMOS-compatible voltages," Nature, vol. 562, pp. 101, 2018.

[201] B. Corcoran, et al., "Ultra-dense optical data transmission over standard fiber with a single chip source", Nature Communications, vol. 11, Article:2568, 2020. DOI:10.1038/s41467-020-16265-x.

[202] X. Xu, et al., "Photonic perceptron based on a Kerr microcomb for scalable high speed optical neural networks", Laser and Photonics Reviews, vol. 14, no. 8, 2000070, 2020. DOI:10.1002/lpor.202000070.

[203] X. Xu, et al., "11 TOPs photonic convolutional accelerator for optical neural networks", Nature, vol.589 (7840) 44-51 (2021). DOI: 10.1038/s41586-020-03063-0.

[204] X Xu et al., "11 TeraFLOPs per second photonic convolutional accelerator for deep learning optical neural networks", arXiv preprint arXiv:2011.07393 (2020).

[205] D. Moss, "11 Tera-FLOP/s photonic convolutional accelerator and deep learning optical neural networks",

[206] Research Square (2021). DOI: https://doi.org/10.21203/rs.3.rs-493347/v1.

[207] D.Moss, "11.0 Tera-FLOP/second photonic convolutional accelerator for deep learning optical neural networks", TechRxiv. Preprint (2020). https://doi.org/10.36227/techrxiv.13238423.v1.

[208] Moss, David. "11 Tera-flop/s Photonic Convolutional Accelerator for Optical Neural Networks." OSF Preprints, 23 Feb. (2021). DOI: $10.31219 /$ osf.io/vqt4s.

[209] Mengxi Tan, X. Xu, J. Wu, T. G. Nguyen, S. T. Chu, B. E. Little, R. Morandotti, A. Mitchell, and David J. Moss, "Photonic Radio Frequency Channelizers based on Kerr Optical Micro-combs", Journal of Semiconductors, Vol. 42, No. 4, 041302 (2021). (ISSN 1674-4926). DOI:10.1088/1674-4926/42/4/041302.

[210] H. Bao, L.Olivieri, M.Rowley, S.T. Chu, B.E. Little, R.Morandotti, D.J. Moss, J.S.T. Gongora, M.Peccianti and A. Pasquazi, "Laser Cavity Solitons and Turing Patterns in Microresonator Filtered Lasers: properties and perspectives", Paper No. LA2035, Paper No. 11672-5, SPIE LASE, SPIE Photonics West, San Francisco CA March 6-11 (2021). DOI:10.1117/12.2576645

[211] Mengxi Tan, X. Xu, J. Wu, A. Boes, T. G. Nguyen, S. T. Chu, B. E. Little, R. Morandotti, A. Mitchell, and David J. Moss, "Advanced microwave signal generation and processing with soliton crystal microcombs", or "Photonic convolutional accelerator and neural network in the Tera-OPs regime based on Kerr microcombs", Paper No. 11689-38, PW21O-OE201-67, Integrated Optics: Devices, Materials, and Technologies XXV, SPIE Photonics West, San Francisco CA March 6-11 (2021). DOI: $10.1117 / 12.2584017$

[212] Mengxi Tan, Bill Corcoran, Xingyuan Xu, Andrew Boes, Jiayang Wu, Thach Nguyen, Sai T. Chu, Brent E. Little, Roberto Morandotti, Arnan Mitchell, and David J. Moss, "Optical data transmission at 40 Terabits/s with a Kerr soliton crystal microcomb", Paper No.11713-8, PW21O-OE803-23, Next-Generation Optical Communication: Components, Sub-Systems, and Systems X, SPIE Photonics West, San Francisco CA March 6-11 (2021). DOI:10.1117/12.2584014

[213] Mengxi Tan, X. Xu, J. Wu, A. Boes, T. G. Nguyen, S. T. Chu, B. E. Little, R. Morandotti, A. Mitchell, and David J. Moss, "RF and microwave photonic, fractional differentiation, integration, and Hilbert transforms based on Kerr micro-combs", Paper No. 11713-16, PW21O-OE803-24, Next-Generation Optical Communication: Components, Sub-Systems, and Systems X, SPIE Photonics West, San Francisco CA March 6-11 (2021). DOI:10.1117/12.2584018 
[214] Mengxi Tan, X. Xu, J. Wu, A. Boes, T. G. Nguyen, S. T. Chu, B. E. Little, R. Morandotti, A. Mitchell, and David J. Moss, "Broadband photonic RF channelizer with 90 channels based on a soliton crystal microcomb", or "Photonic microwave and RF channelizers based on Kerr micro-combs", Paper No. 11685-22, PW21O-OE106-49, Terahertz, RF, Millimeter, and Submillimeter-Wave Technology and Applications XIV, SPIE Photonics West, San Francisco CA March 6-11 (2021). DOI: $10.1117 / 12.2584015$

[215] X. Xu, M. Tan, J. Wu, S. T. Chu, B. E. Little, R. Morandotti, A. Mitchell, B. Corcoran, D. Hicks, and D. J. Moss, "Photonic perceptron based on a Kerr microcomb for scalable high speed optical neural networks", IEEE Topical Meeting on Microwave Photonics (MPW), pp. 220-224, Matsue, Japan, November 24-26, 2020. Electronic ISBN:978-4-88552-331-1. DOI: 10.23919/MWP48676.2020.9314409

[216] Mengxi Tan, Bill Corcoran, Xingyuan Xu, Andrew Boes, Jiayang Wu, Thach Nguyen, S.T. Chu, B. E. Little, Roberto Morandotti, Arnan Mitchell, and David J. Moss, "Ultra-high bandwidth optical data transmission with a microcomb", IEEE Topical Meeting on Microwave Photonics (MPW), pp. 78-82. Virtual Conf., Matsue, Japan, November 24-26, 2020. Electronic ISBN:978-488552-331-1. DOI: 10.23919/MWP48676.2020.9314476

[217] M. Tan, X. Xu, J. Wu, R. Morandotti, A. Mitchell, and D. J. Moss, "RF and microwave high bandwidth signal processing based on Kerr Micro-combs", Advances in Physics X, VOL. 6, NO. 1, 1838946 (2020). DOI:10.1080/23746149.2020.1838946.

[218] M Tan, X Xu, J Wu, DJ Moss, "High bandwidth temporal RF photonic signal processing with Kerr micro-combs: integration, fractional differentiation and Hilbert transforms", arXiv preprint arXiv:2103.03674 (2021).

[219] D. Moss, "Temporal RF photonic signal processing with Kerr micro-combs: Hilbert transforms, integration and fractional differentiation", OSF Preprints, 18 Feb. (2021). DOI: 10.31219/osf.io/hx9gb.

[220] D. Moss, "RF and microwave photonic high bandwidth signal processing based on Kerr micro-comb sources", TechRxiv. Preprint (2020). DOI:10.36227/techrxiv.12665609.v3.

[221] D. Moss, "RF and microwave photonic signal processing with Kerr micro-combs", Research Square (2021). DOI: 10.21203/rs.3.rs-473364/v1.

[222] M.Tan, X. Xu, J. Wu, D.J. Moss, "RF Photonic Signal Processing with Kerr Micro-Combs: Integration, Fractional Differentiation and Hilbert Transforms", Preprints (2020). 2020090597. doi:10.20944/preprints202009.0597.v1.

[223] Mengxi Tan, Xingyuan Xu, Jiayang Wu, Thach G. Nguyen, Sai T. Chu, Brent E. Little, Roberto Morandotti, Arnan Mitchell, and David J. Moss, "Photonic Radio Frequency Channelizers based on Kerr Micro-combs and Integrated Micro-ring Resonators", JOSarXiv.202010.0002.

[224] Mengxi Tan, Xingyuan Xu, David Moss "Tunable Broadband RF Photonic Fractional Hilbert Transformer Based on a Soliton Crystal Microcomb", Preprints, DOI: 10.20944/preprints202104.0162.v1

[225] Mengxi Tan, X. Xu, J. Wu, T. G. Nguyen, S. T. Chu, B. E. Little, R. Morandotti, A. Mitchell, and David J. Moss, "Orthogonally polarized Photonic Radio Frequency single sideband generation with integrated micro-ring resonators", Journal of Semiconductors vol. 42, No.4, 041305 (2021). DOI: 10.1088/1674-4926/42/4/041305.

[226] Mengxi Tan, X. Xu, J. Wu, T. G. Nguyen, S. T. Chu, B. E. Little, R. Morandotti, A. Mitchell, and David J. Moss, "Photonic Radio Frequency Channelizers based on Kerr Optical Micro-combs", Journal of Semiconductors 42 (4), 041302 (2021). (ISSN 1674-4926). DOI:10.1088/1674-4926/42/4/041302.

[227] Mengxi Tan, Xingyuan Xu, David Moss "Tunable Broadband RF Photonic Fractional Hilbert Transformer Based on a Soliton Crystal Microcomb", Preprints, DOI: 10.20944/preprints202104.0162.v1

[228] Mengxi Tan, X. Xu, J. Wu, T. G. Nguyen, S. T. Chu, B. E. Little, R. Morandotti, A. Mitchell, and David J. Moss, "Orthogonally polarized Photonic Radio Frequency single sideband generation with integrated micro-ring resonators", Journal of Semiconductors 42 (4), 041305 (2021). DOI: 10.1088/1674-4926/42/4/041305.

[229] L. Razzari, D. Duchesne, M. Ferrera, et al., "CMOS-compatible integrated optical hyper-parametric oscillator," Nature Photonics, vol. 4, no. 1, pp. 41-45 (2010).

[230] M. Ferrera, L. Razzari, D. Duchesne, et al., "Low-power continuous-wave nonlinear optics in doped silica glass integrated waveguide structures," Nature Photonics, vol. 2, no. 12, pp. 737-740 (2008).

[231] A. Pasquazi, et al., "Sub-picosecond phase-sensitive optical pulse characterization on a chip", Nature Photonics, vol. 5, no. 10, pp. 618-623 (2011). DOI: 10.1038/nphoton.2011.199.

[232] D. Duchesne, M. Peccianti, M. R. E. Lamont, et al., "Supercontinuum generation in a high index doped silica glass spiral waveguide," Optics Express, vol. 18, no, 2, pp. 923-930 (2010).

[233] M. Ferrera, et al., "On-chip CMOS-compatible all-optical integrator", Nature Communications, vol. 1, Article 29 (2010).

[234] H. Bao et al., "Turing patterns in a fibre laser with a nested micro-resonator: robust and controllable micro-comb generation", Physical Review Research, vol. 2, pp. 023395 (2020).

[235] L. D. Lauro, J. Li, D. J. Moss, R. Morandotti, S. T. Chu, M. Peccianti, and A. Pasquazi, "Parametric control of thermal selfpulsation in micro-cavities," Opt. Lett. vol. 42, no. 17, pp. 3407-3410, Aug. 2017.

[236] H. Bao et al., "Type-II micro-comb generation in a filter-driven four wave mixing laser," Photonics Research, vol. 6, no. 5, pp. B67-B73 (2018).

[237] A. Pasquazi, et al., "All-optical wavelength conversion in an integrated ring resonator," Optics Express, vol. 18, no. 4, pp. 38583863 (2010).

[238] A. Pasquazi, Y. Park, J. Azana, et al., "Efficient wavelength conversion and net parametric gain via Four Wave Mixing in a high index doped silica waveguide," Optics Express, vol. 18, no. 8, pp. 7634-7641 (2010). 
[239] M. Peccianti, M. Ferrera, L. Razzari, et al., "Subpicosecond optical pulse compression via an integrated nonlinear chirper," Optics Express, vol. 18, no. 8, pp. 7625-7633 (2010).

[240] D. Duchesne, M. Ferrera, L. Razzari, et al., "Efficient self-phase modulation in low loss, high index doped silica glass integrated waveguides," Optics Express, vol. 17, no. 3, pp. 1865-1870 (2009).

[241] M. Peccianti, et al., "Demonstration of an ultrafast nonlinear microcavity modelocked laser", Nature Communications, vol. 3, pp. 765 (2012).

[242] M. Kues, et al., "Passively modelocked laser with an ultra-narrow spectral width", Nature Photonics, vol. 11, no. 3, pp. 159 (2017). DOI:10.1038/nphoton.2016.271

[243] A. Pasquazi, L. Caspani, M. Peccianti, et al., "Self-locked optical parametric oscillation in a CMOS compatible microring resonator: a route to robust optical frequency comb generation on a chip," Optics Express, vol. 21, no. 11, pp. 13333-13341 (2013).

[244] A. Pasquazi, M. Peccianti, B. E. Little, et al., "Stable, dual mode, high repetition rate mode-locked laser based on a microring resonator," Optics Express, vol. 20, no. 24, pp. 27355-27362 (2012).

[245] C. Reimer, L. Caspani, M. Clerici, et al., "Integrated frequency comb source of heralded single photons," Optics Express, vol. 22, no. 6, pp. 6535-6546 (2014).

[246] C. Reimer, et al., "Cross-polarized photon-pair generation and bi-chromatically pumped optical parametric oscillation on a chip", Nature Communications, vol. 6, Article 8236 (2015). DOI: 10.1038/ncomms9236

[247] L. Caspani, C. Reimer, M. Kues, et al., "Multifrequency sources of quantum correlated photon pairs on-chip: a path toward integrated Quantum Frequency Combs," Nanophotonics, vol. 5, no. 2, pp. 351-362 (2016).

[248] C. Reimer, M. Kues, P. Roztocki, B. Wetzel, F. Grazioso, B. E. Little, S. T. Chu, T. Johnston, Y. Bromberg, L. Caspani, D. J. Moss, and R. Morandotti, "Generation of multiphoton entangled quantum states by means of integrated frequency combs," Science, vol. 351, no. 6278, pp. 1176-1180 (2016).

[249] P. Roztocki, M. Kues, C. Reimer, B. Wetzel, S. Sciara, Y. Zhang, A. Cino, B. E. Little, S. T. Chu, D. J. Moss, and R. Morandotti, "Practical system for the generation of pulsed quantum frequency combs," Optics Express, vol. 25, no. 16, pp. 18940-18949 (2017).

[250] Y. Zhang, et al., "Induced photon correlations through superposition of two four-wave mixing processes in integrated cavities", Laser and Photonics Reviews, vol. 14, no. 7, 2000128 (2020). DOI: 10.1002/lpor.202000128

[251] M. Kues, C. Reimer, A. Weiner, J. Lukens, W. Munro, D. J. Moss, and R. Morandotti, "Quantum Optical Micro-combs", Nature Photonics, vol. 13, no.3, pp. 170-179 (2019).

[252] C. Reimer, et al.,"High-dimensional one-way quantum processing implemented on d-level cluster states", Nature Physics, vol. 15, no.2, pp. 148-153 (2019).

[253] T.Ido, H.Sano, D.J.Moss, S.Tanaka, and A.Takai, "Strained InGaAs/InAlAs MQW electroabsorption modulators with large bandwidth and low driving voltage", Photonics Technology Letters, Vol. 6, 1207 (1994). DOI: 10.1109/68.329640.

[254] H. Arianfard et al., "Three waveguide coupled sagnac loop reflectors for advanced spectral engineering," J. Lightwave Technol., doi: 10.1109/JLT.2021.3066256.

[255] J. Wu et al., "Nested configuration of silicon microring resonator with multiple coupling regimes," IEEE Photon. Technol. Lett., vol. 25, no. 6, pp. 580-583, Mar. 2013.

[256] J. Wu, T. Moein, X. Xu, and D. J. Moss, "Advanced photonic filters based on cascaded Sagnac loop reflector resonators in silicon-on-insulator nanowires,” APL Photonics, vol. 3, 046102 (2018). DOI:/10.1063/1.5025833Apr. 2018.

[257] J Wu, T Moein, X Xu, DJ Moss, "Silicon photonic filters based on cascaded Sagnac loop resonators", arXiv preprint arXiv: 1805.05405a (2018).

[258] J. Wu, T. Moein, X. Xu, G. H. Ren, A. Mitchell, and D. J. Moss, "Micro-ring resonator quality factor enhancement via an integrated Fabry-Perot cavity," APL Photonics, vol. 2, 056103 (2017).

[259] H. Arianfard, J. Wu, S. Juodkazis, and D. J. Moss, "Advanced Multi-Functional Integrated Photonic Filters Based on Coupled Sagnac Loop Reflectors", Journal of Lightwave Technology, Vol. 39, No.5, pp.1400-1408 (2021). DOI: 10.1109/JLT.2020.3037559.

[260] David J. Moss, "Optimization of Optical Filters based on Integrated Coupled Sagnac Loop Reflectors", Research Square (2021). DOI: https://doi.org/10.21203/rs.3.rs-478204/v1

[261] H. Arianfard, J. Wu, S. Juodkazis, D. J. Moss, "Spectral Shaping Based on Integrated Coupled Sagnac Loop Reflectors Formed by a Self-Coupled Wire Waveguide", submitted, IEEE Photonics Technology Letters, vol. 33 (2021).

[262] J. Wu et al., "Graphene oxide waveguide and micro-ring resonator polarizers," Laser Photonics Rev., vol. 13, no. 9, pp. 1900056, Aug. 2019.

[263] Y. Zhang et al., "Optimizing the Kerr nonlinear optical performance of silicon waveguides integrated with 2D graphene oxide films,” J. Lightwave Technol., doi: 10.1109/JLT.2021.3069733.

[264] Y. Qu et al., "Analysis of four-wave mixing in silicon nitride waveguides integrated with 2D layered graphene oxide films," J. Lightwave Technol., vol. 39, no. 9, pp. 2902-2910, May. 2021.

[265] Y. Qu et al., "Enhanced four-wave mixing in silicon nitride waveguides integrated with 2D layered graphene oxide films," Adv. Opt. Mater., vol. 8, no. 20, pp. 2001048, Oct. 2020.

[266] J. Wu et al., "2D layered graphene oxide films integrated with micro-ring resonators for enhanced nonlinear optics," Small, vol. 16, no. 16, pp. 1906563, Mar. 2020. 
[267] Moss, David; Wu, Jiayang; xu, xingyuan; Yang, Yunyi; jia, linnan; Zhang, Yuning; et al. (2020): Enhanced optical four-wavemixing in integrated ring resonators with graphene oxide films. TechRxiv. Preprint. https://doi.org/10.36227/techrxiv.11859429.v1.

[268] Wu, J.; Yang, Y.; Qu, Y.; Jia, L.; Zhang, Y.; Xu, X.; Chu, S.T.; Little, B.E.; Morandotti, R.; Jia, B.; Moss, D.J. Enhancing Third Order Nonlinear Optics in Integrated Ring Resonators with 2D Material Films. Preprints 2020, 2020030107

[269] Wu, J.; Yang, Y.; Qu, Y.; Jia, L.; Zhang, Y.; Xu, X.; Chu, S.T.; Little, B.E.; Morandotti, R.; Jia, B.; Moss, D.J. Enhancing Third Order Nonlinear Optics in Integrated Ring Resonators with 2D Material Films. Preprints 2020, 2020030107

[270] J Wu et al., "Enhanced four-wave-mixing with 2D layered graphene oxide films integrated with CMOS compatible micro-ring resonators", arXiv preprint, arXiv:2002.04158 (2020).

[271] Y. Zhang et al., "Enhanced Kerr nonlinearity and nonlinear figure of merit in silicon nanowires integrated with 2D graphene oxide films," ACS Appl. Mater. Interfaces, vol. 12, no. 29, pp. 33094-33103, Jun. (2020).

[272] Y Zhang et al., "Enhanced nonlinear optical figure-of-merit at $1550 \mathrm{~nm}$ for silicon nanowires integrated with graphene oxide layered films", arXiv preprint arXiv:2004.08043 (2020).

[273] D. Moss et al., "Transforming silicon into a high performing integrated nonlinear photonics platform by integration with $2 \mathrm{D}$ graphene oxide films", TechRxiv. Preprint. (2020). https://doi.org/10.36227/techrxiv.12061809.v1.

[274] D. Moss, "Elevating silicon into a high performance nonlinear optical platform through the integration of 2D graphene oxide thin films", Research Square (2021). DOI: https://doi.org/10.21203/rs.3.rs-511259/v1.

[275] Moss, D.; Wu, J.; Jia, B.; Yang, Y.; Qu, Y.; Jia, L.; Zhang, Y. "Improved Nonlinear Optics in Silicon-on-insulator Nanowires Integrated with 2D Graphene Oxide Films", Preprints (2020), 2020040033.

[276] Y. Yang et al., "Invited article: enhanced four-wave mixing in waveguides integrated with graphene oxide," APL Photonics, vol. 3, no. 12, pp. 120803 , Oct. 2018.

[277] TD Vo et al., "Silicon-chip-based real-time dispersion monitoring for $640 \mathrm{Gbit} / \mathrm{s}$ DPSK signals", Journal of Lightwave Technology, vol. 29, no. 12, 1790-1796 (2011). 


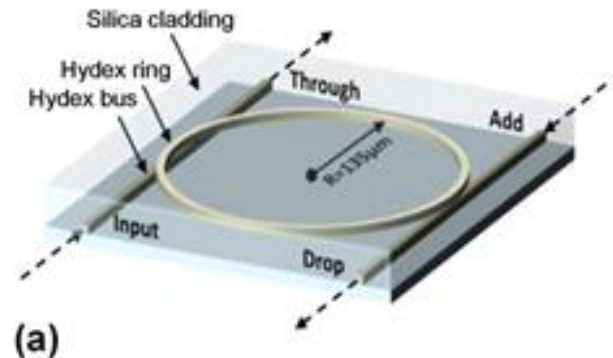

(a)

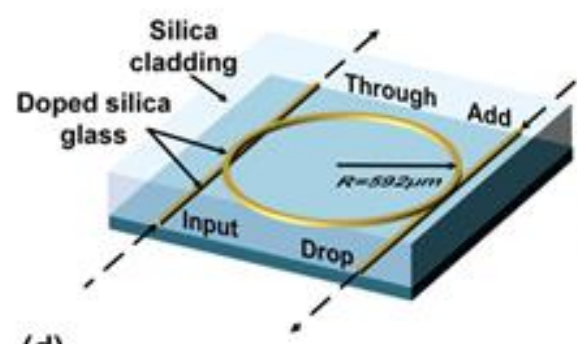

(d)

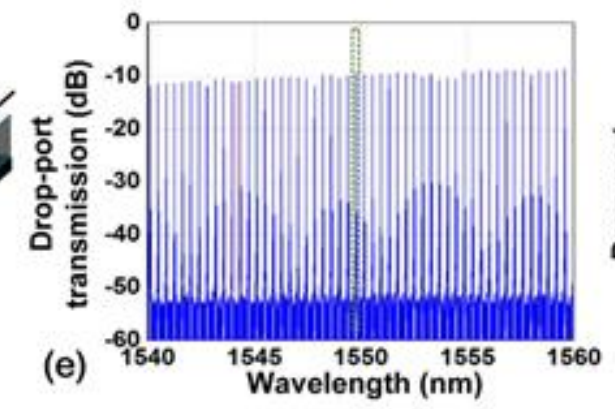

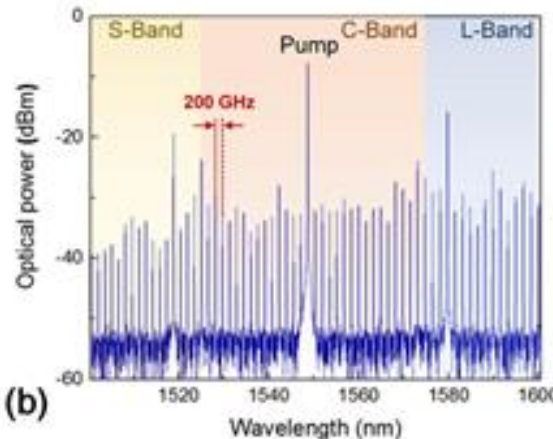
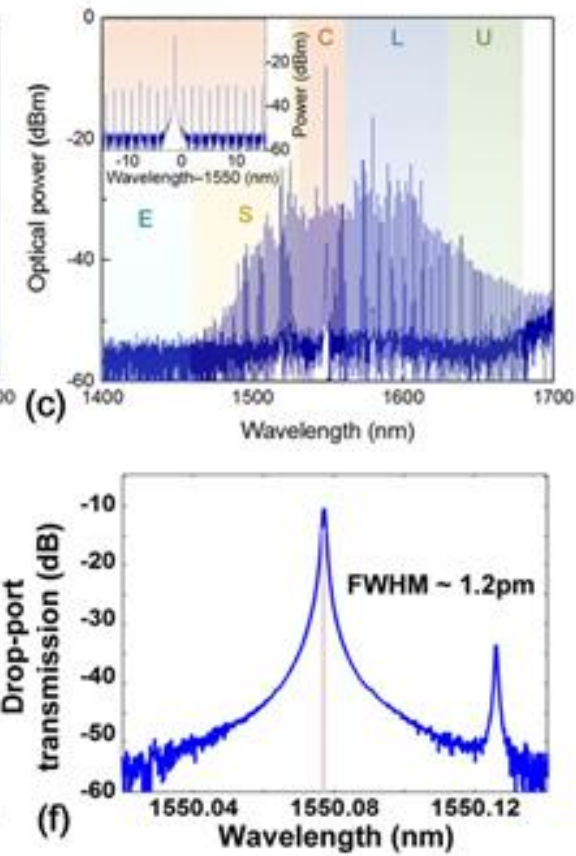

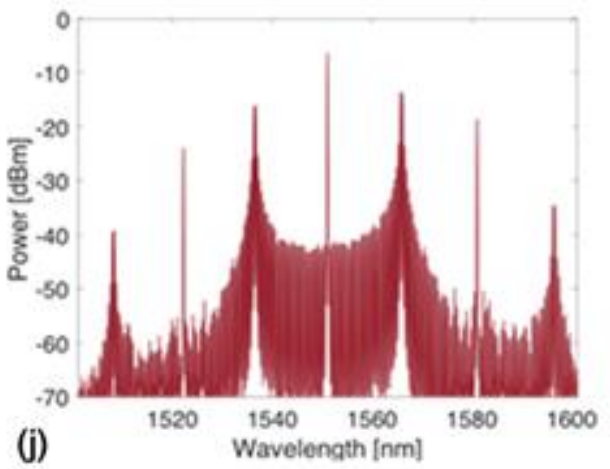

Fig. 1. Schematic illustration of the integrated MRRs for generating the Kerr micro-comb for both (a-c) 200GHz FSR combs and (d-j) 49GHz combs. (b, c) Optical spectra of the micro-combs generated by 200GHz MRR with a span of (b) $100 \mathrm{~nm}$ and (c) 300 nm. (j) Optical spectra of the micro-combs generated by $50 \mathrm{GHz}$ MRR with a span of $100 \mathrm{~nm}$. (e) Drop-port transmission spectrum of the integrated MRR with a span of $5 \mathrm{~nm}$, showing an optical free spectral range of $49 \mathrm{GHz}$. (f) A resonance at $193.294 \mathrm{THz}$ with full width at half maximum (FWHM) of $124.94 \mathrm{MHz}$, corresponding to a quality factor of $1.549 \times 10^{6}$.

\section{Figure 1}



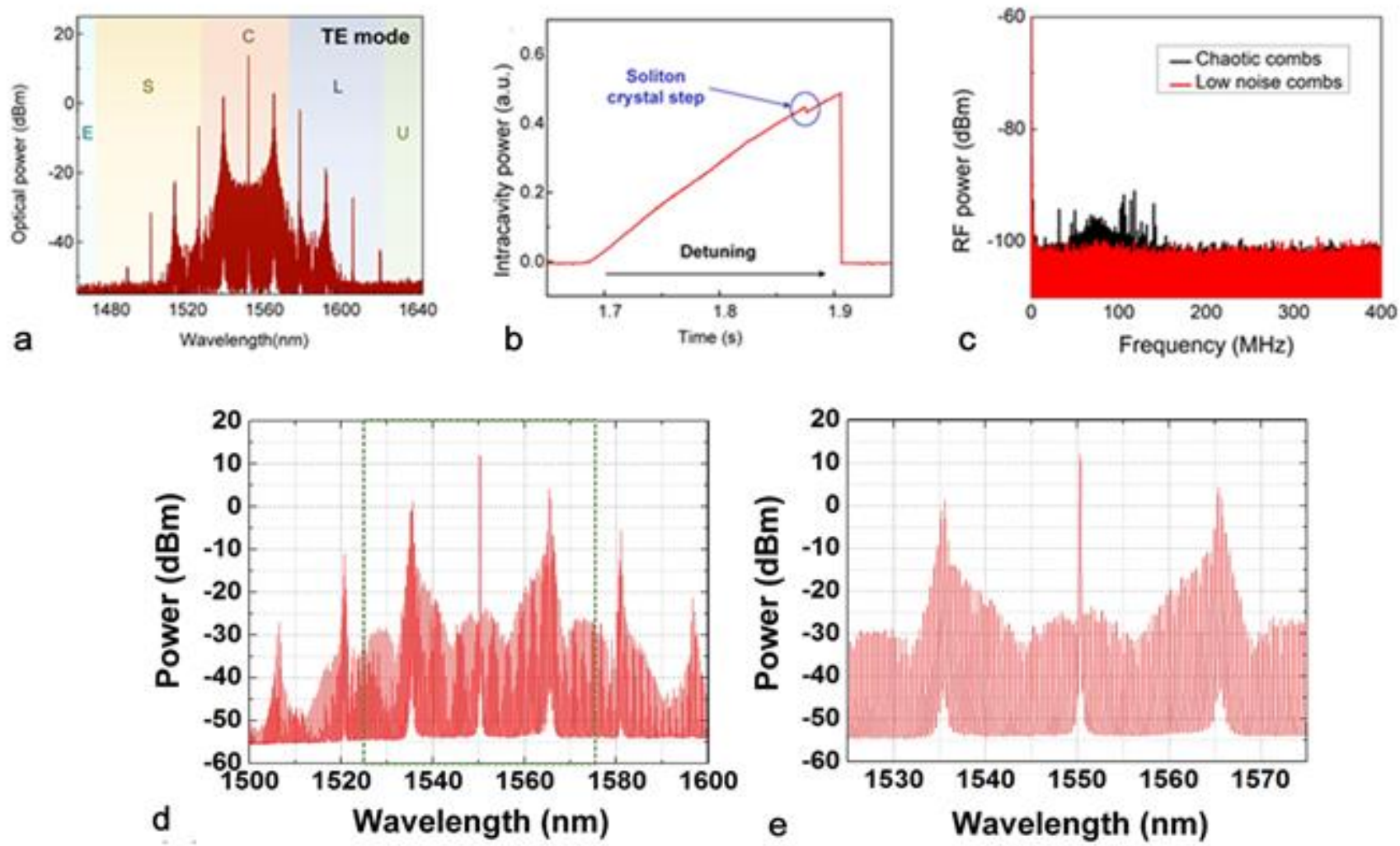

Fig. 2. (a, d, e) Optical spectra of various soliton crystal micro-combs. (b) Optical power output versus pump tuning, showing the very small power jump at the onset of soliton crystal combs. (c) Transition from high RF noise chaotic state to low noise state of the soliton crystal comb. 

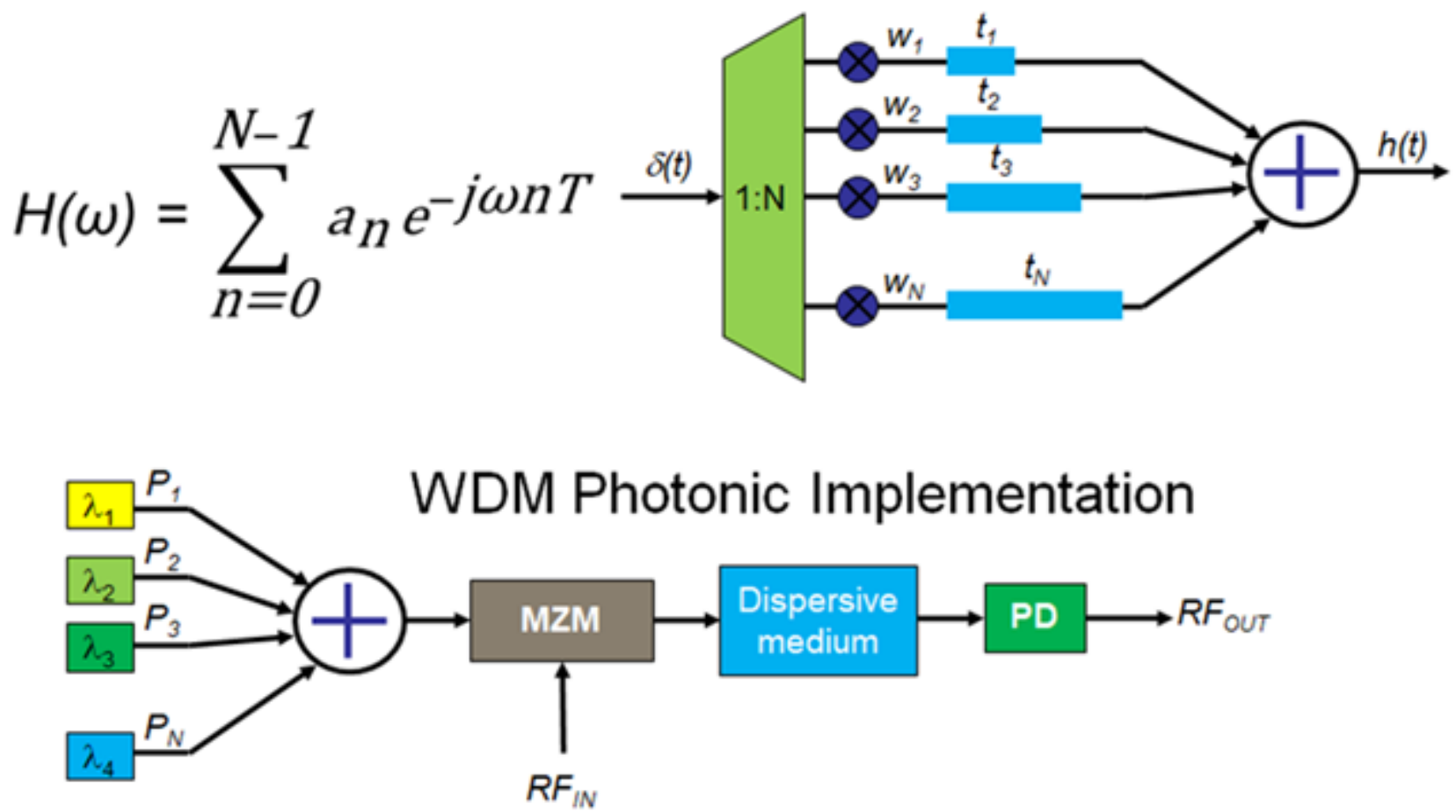

Fig. 3. Theoretical schematic of the principle of transversal filters using wavelength multiplexing. MZM: Mach-Zehnder modulators. PD: photo-detector.

Figure 3 

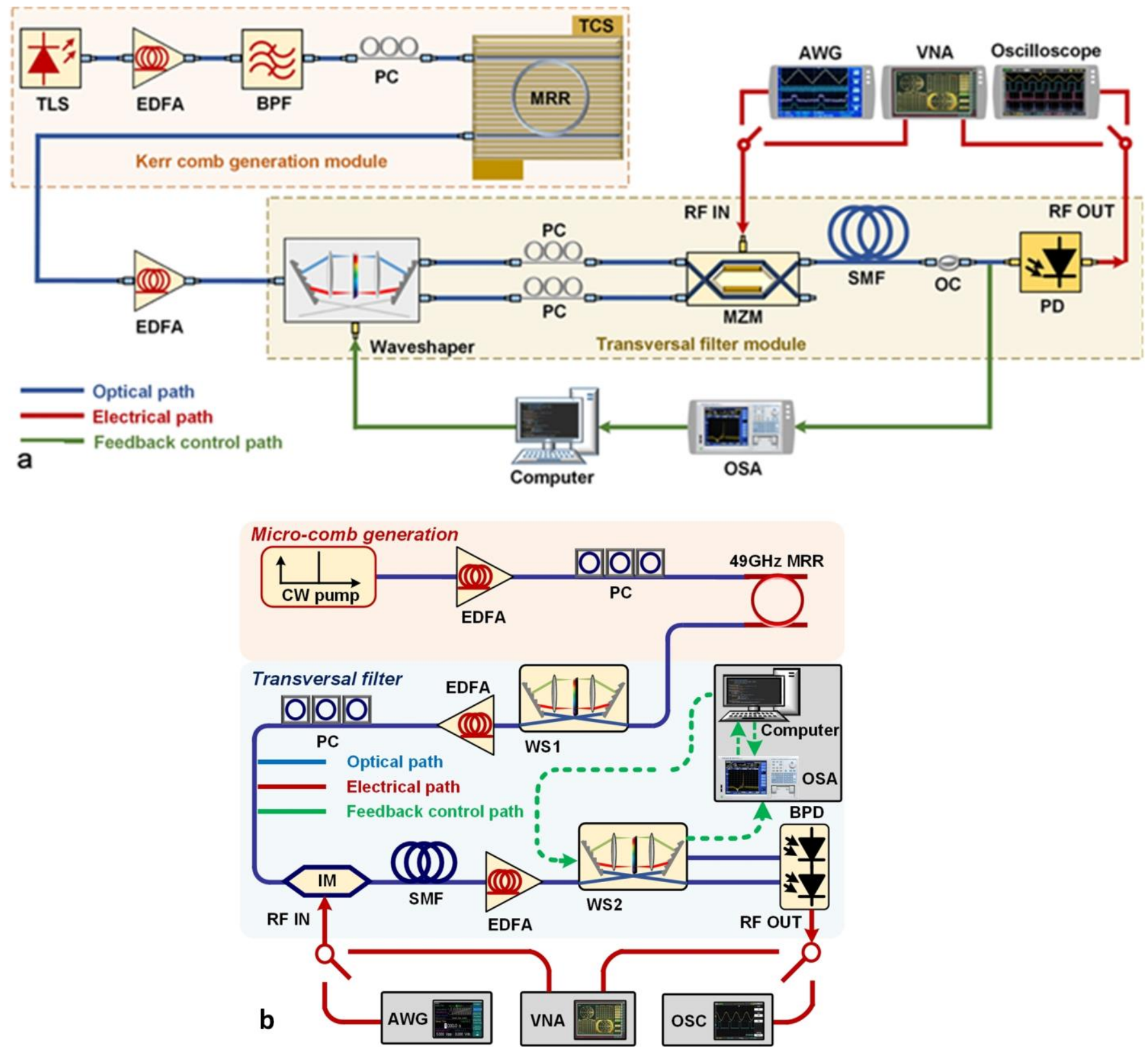

Fig. 4. Experimental schematic for RF transversal filters based on $200 \mathrm{GHz}$ microcomb (top) and $49 \mathrm{GHz}$ microcomb (bottom). TLS: tunable laser source. EDFA: erbium-doped fiber amplifier. PC: polarization controller. BPF: optical bandpass filter. TCS: temperature control stage. MRR: micro-ring resonator. WS: WaveShaper. OC: optical coupler. SMF: single mode fibre. OSA: optical spectrum analyzer. AWG: arbitrary waveform generator. VNA: vector network analyser. PD: photodetector. BPD: balanced photodetector. 

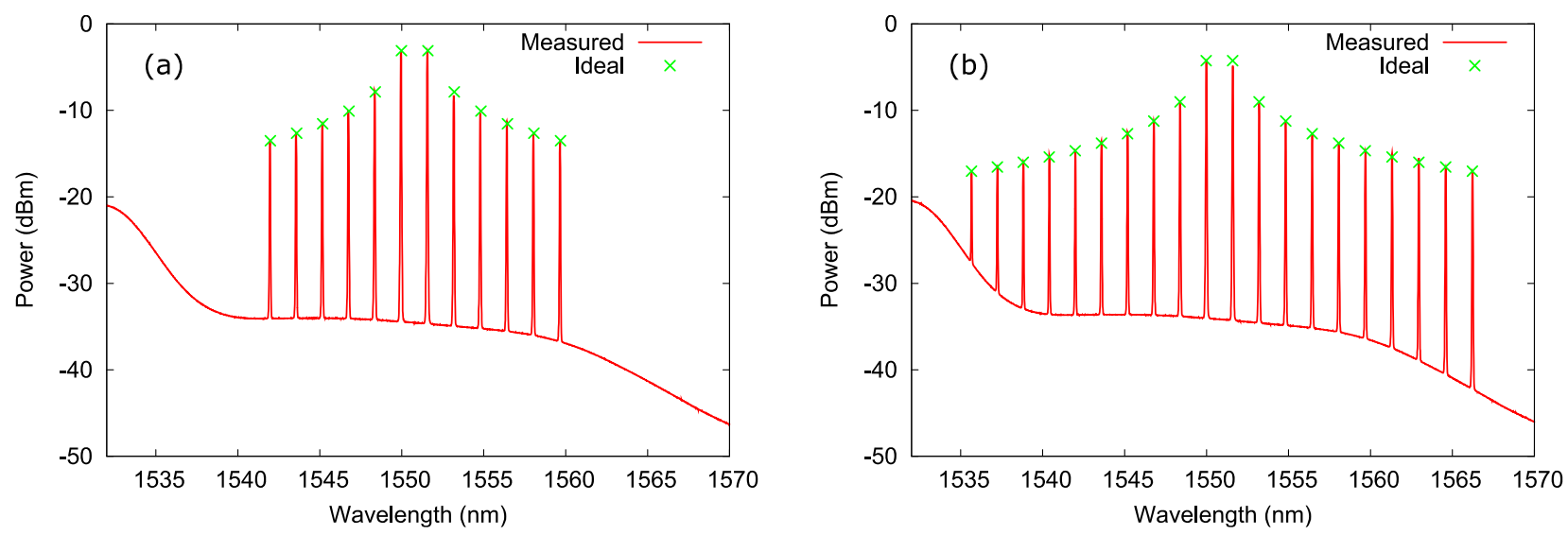

Fig. 5. Shaped optical spectra showing the weight of each tap for: (a) 12 tap filter, and (b) 20 tap filter

Figure 5 

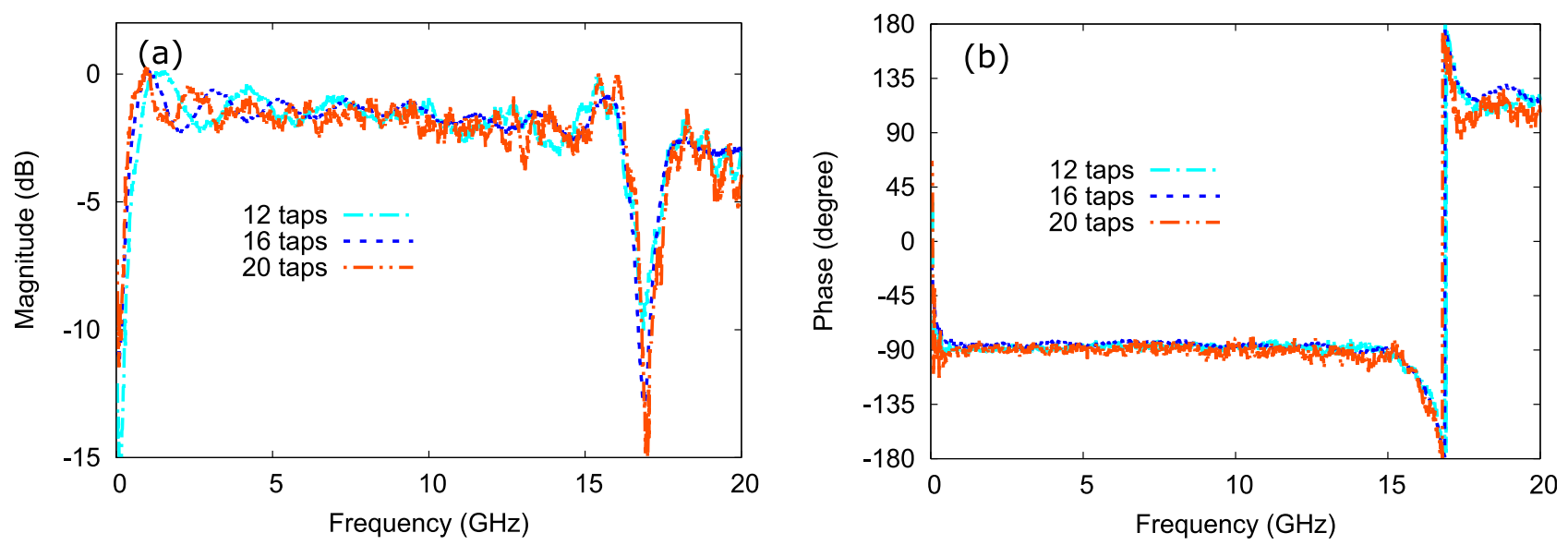

Fig. 6. Measured system RF frequency response for different number of filter taps: (a) amplitude; and (b) phase response.

\section{Figure 6}




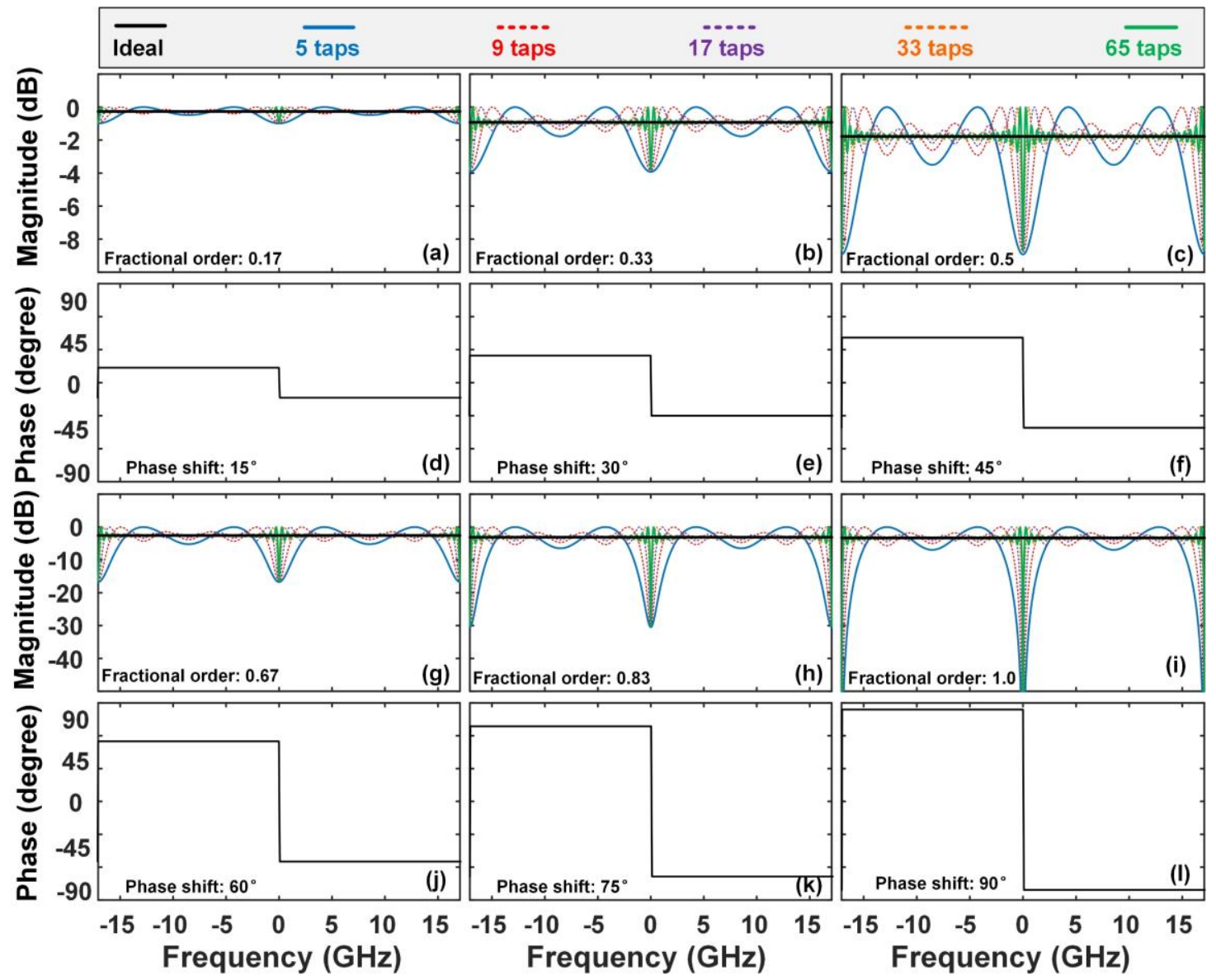

Fig. 7. Theoretical RF amplitude and phase response of FHTs with (a, d) $15^{\circ}$, (b, e) $30^{\circ}$, (c, f) $45^{\circ}$, (g, j) $60^{\circ}$, (h, k) $75^{\circ}$, and (i, l) $90^{\circ}$ phase shifts. The amplitude of the fractional Hilbert transformers designed based on Eq. (3) (colour curves) are shown according to the number of taps employed.

(Maybe separate the top and bottom parts a bit) 


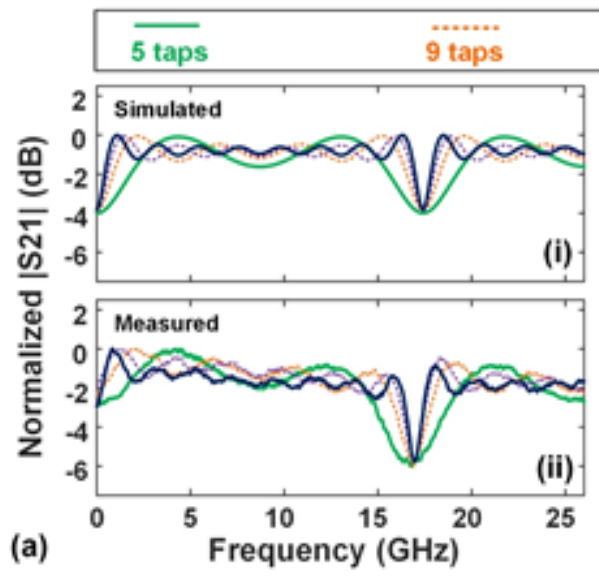

(i).
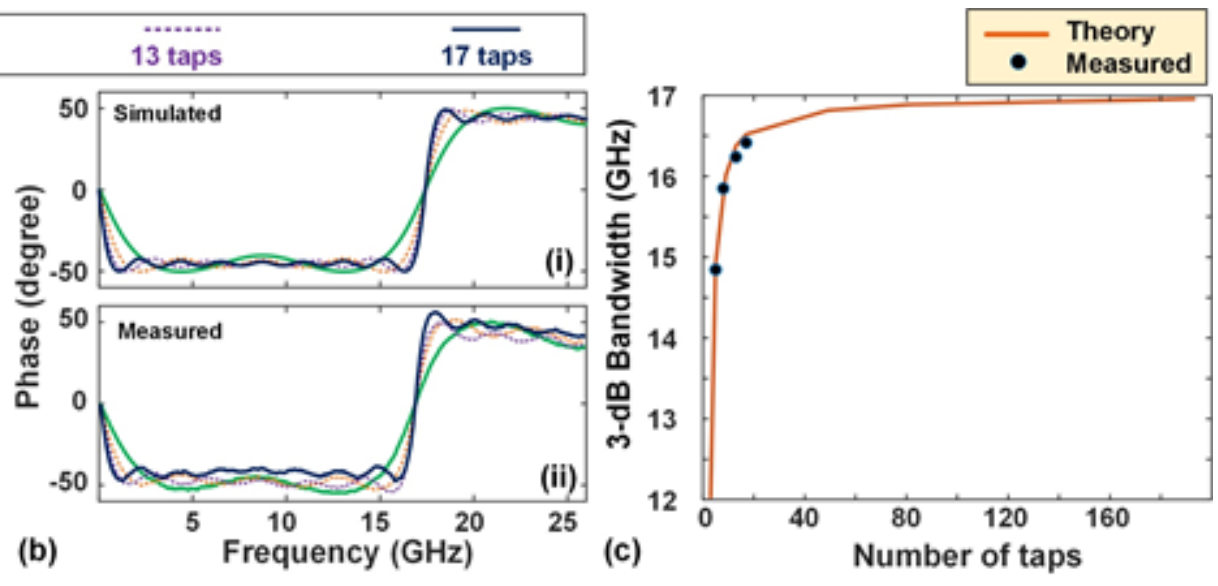

Fig. 8. (a) and (b) Simulated and measured amplitude and phase response for the FHT for different numbers of taps for a FHT phase shift of $45^{\circ}$. (c) Simulated and experimental results of 3-dB bandwidth with different taps for a phase shift of $45^{\circ}$.

Separate figure c into its own figure

Figure 8 

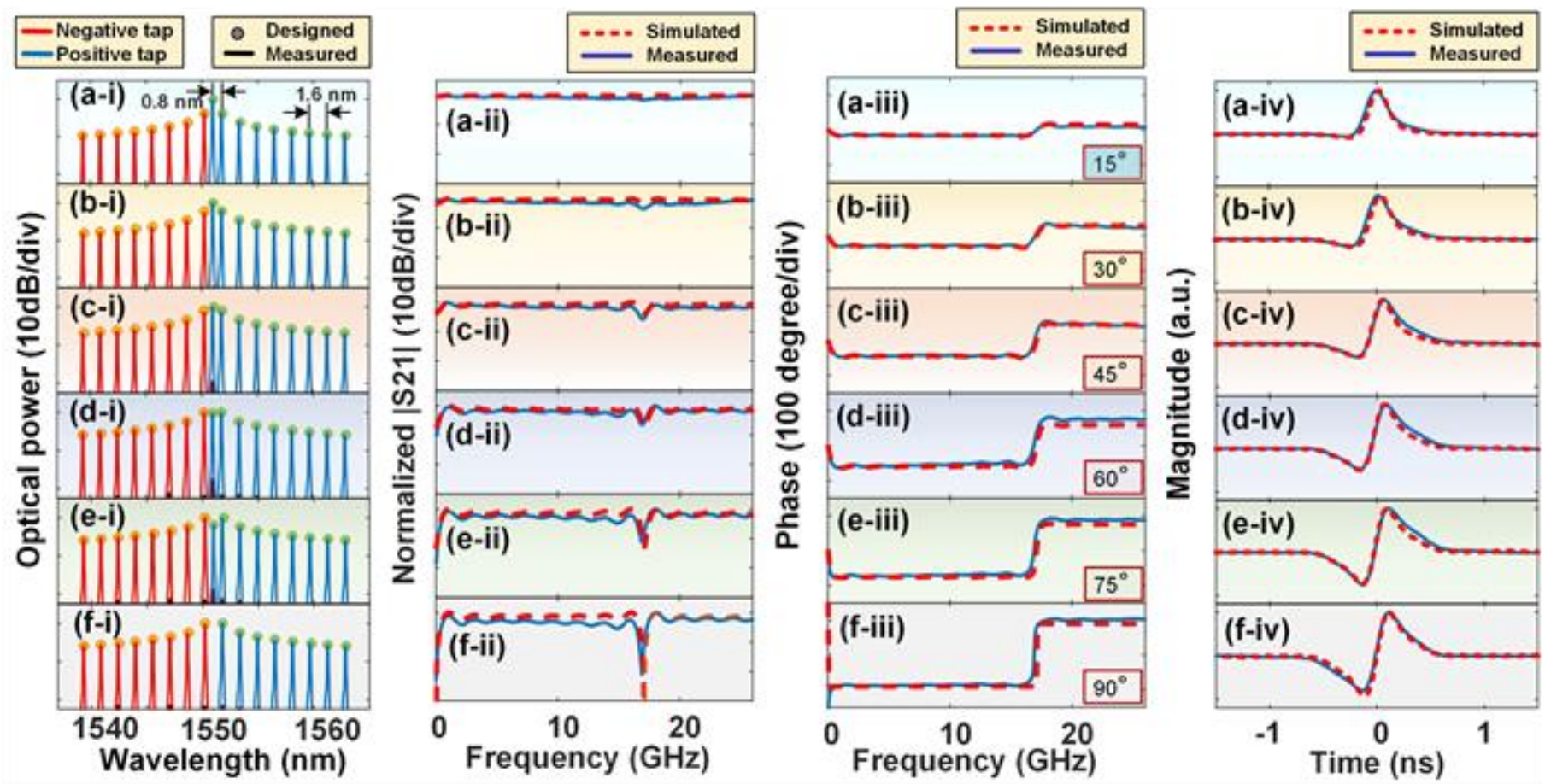

Fig. 9. Simulated (dashed curves) and experimental (solid curves) results of FHT with various phase shifts of (a) $15^{\circ}$, (b) $30^{\circ}$, (c) $45^{\circ}$, (d) $60^{\circ}$, (e) $75^{\circ}$, and (f) $90^{\circ}$. (i) Optical spectra of the shaped micro-comb corresponding with positive and negative tap weights (ii) RF amplitude responses with fractional orders of $0.166,0.333,0.5,0.667,0.833$, and 1. (iii) RF phase responses with phase shifts of $15^{\circ}, 30^{\circ}, 45^{\circ}, 60^{\circ}, 75^{\circ}$ and $90^{\circ}$. (iv) Output temporal intensity waveforms after the FHT.

\section{Figure 9}



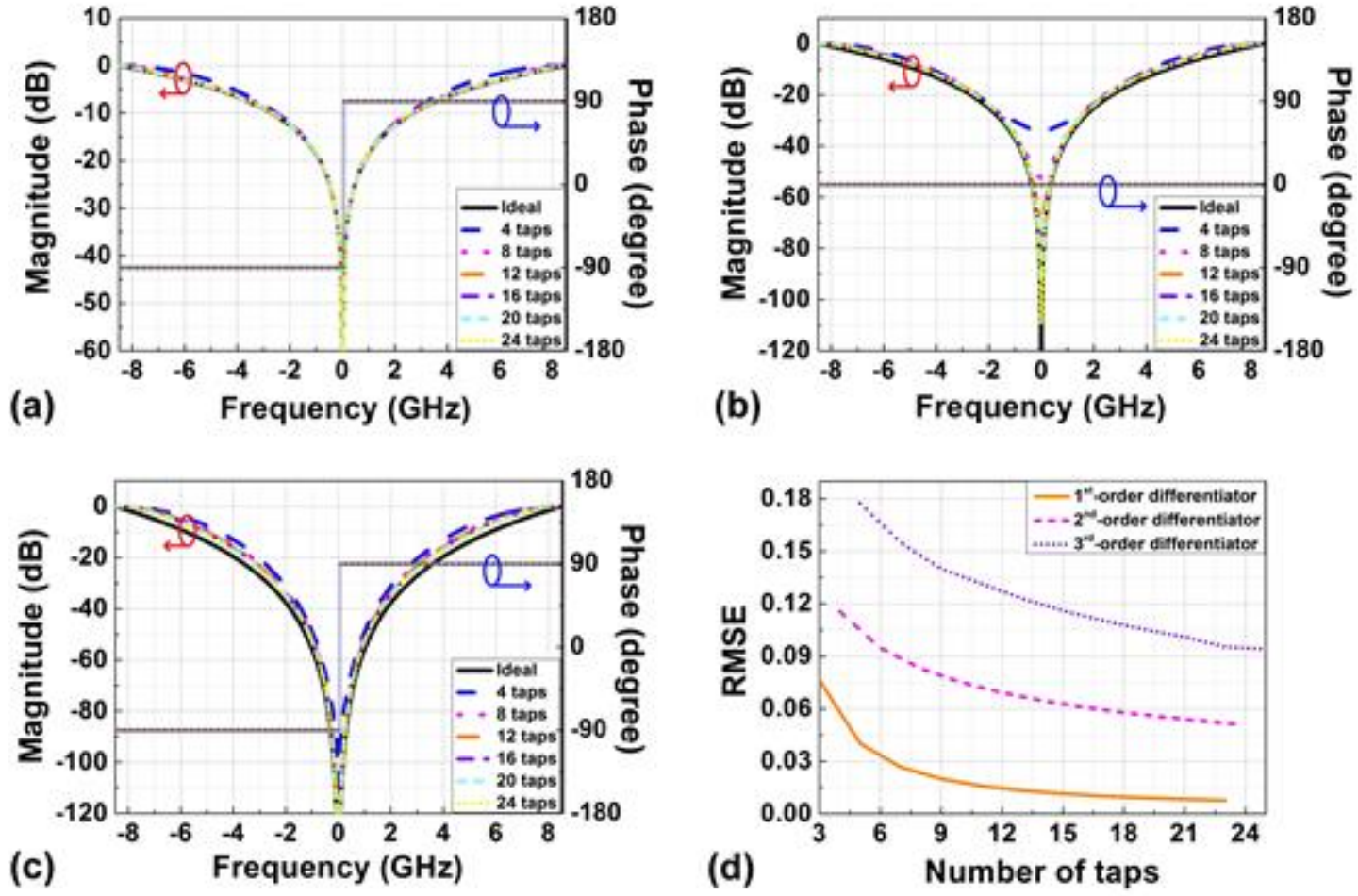

Fig. 10. Simulated RF amplitude and phase responses of the (a) first-, (b) second-, and (c) third-order temporal differentiators. (d) RMSEs between calculated and ideal RF amplitude responses of the first-, second-, and third-order intensity differentiators as a function of the number of taps. 

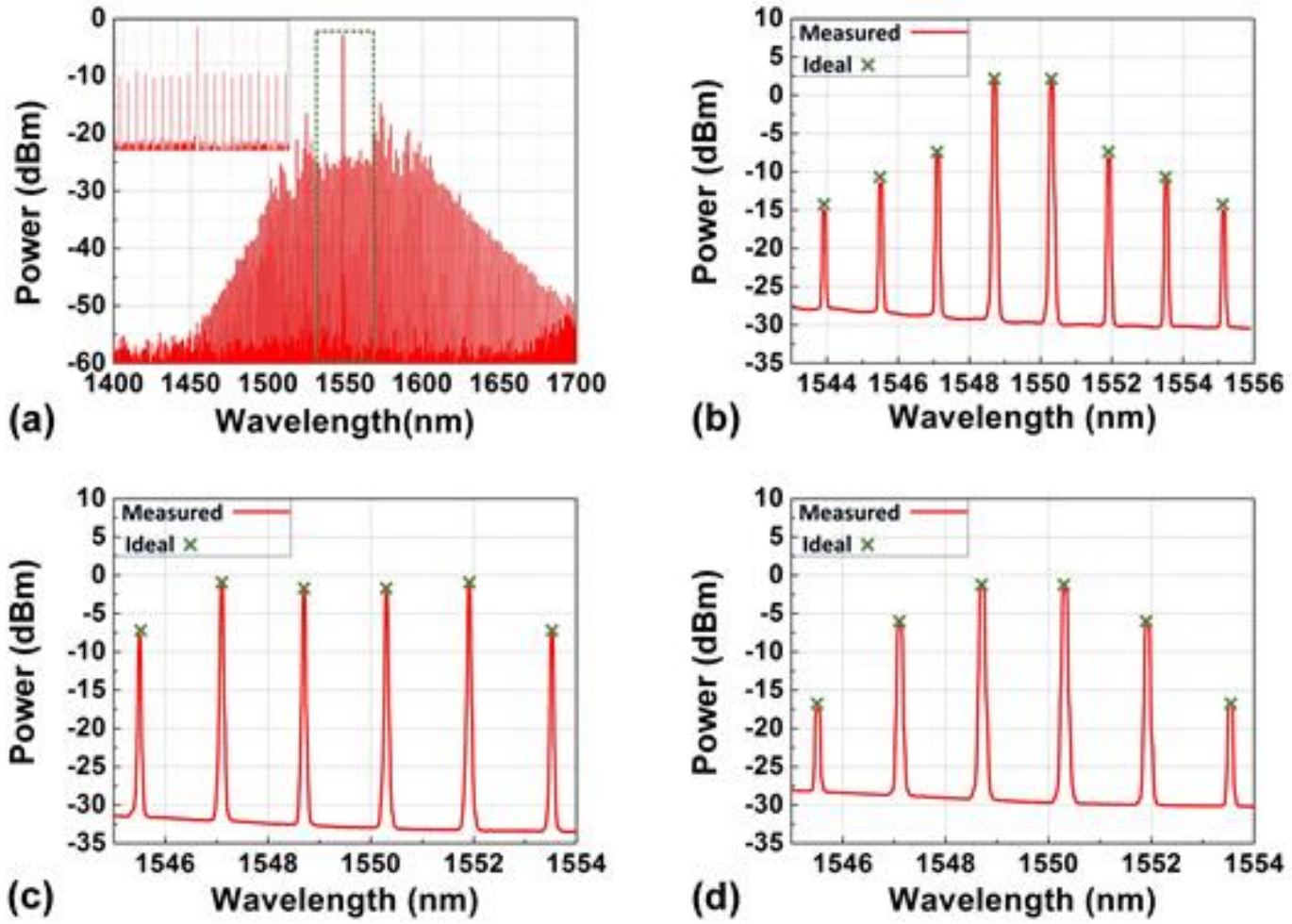

Fig. 11. (a) Optical spectrum of the generated Kerr comb in a 300-nm wavelength range. Inset shows a zoom-in spectrum with a span of $\sim 32 \mathrm{~nm}$. (b)-(d) Measured optical spectra (red solid) of the shaped optical combs and ideal tap weights (green crossing) for the first-, second-, and third-order intensity differentiators. 

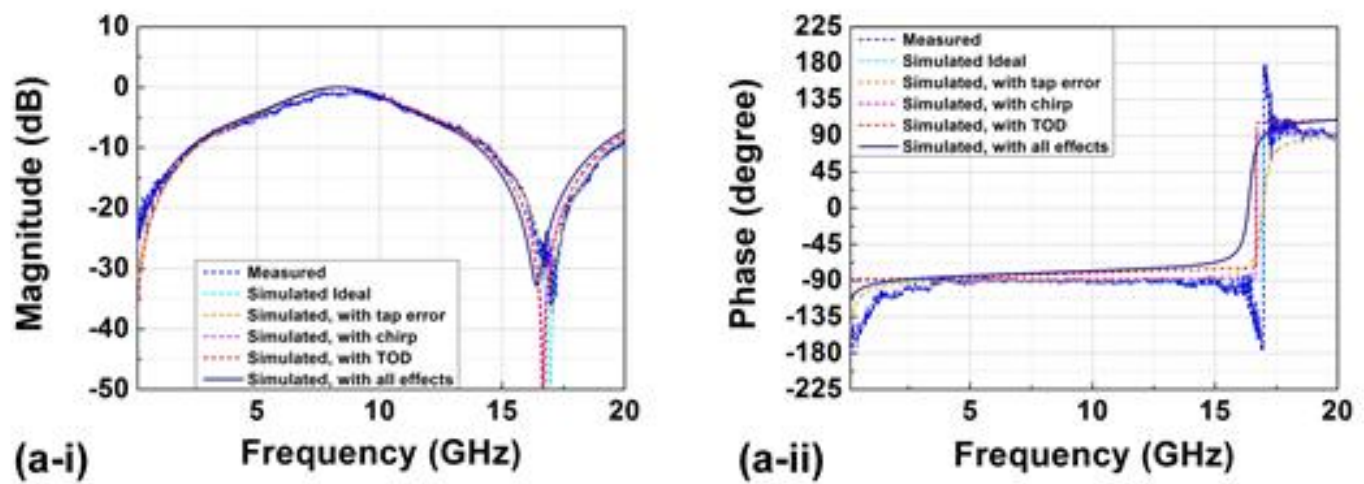

(a-i)

Frequency $(\mathrm{GHz})$
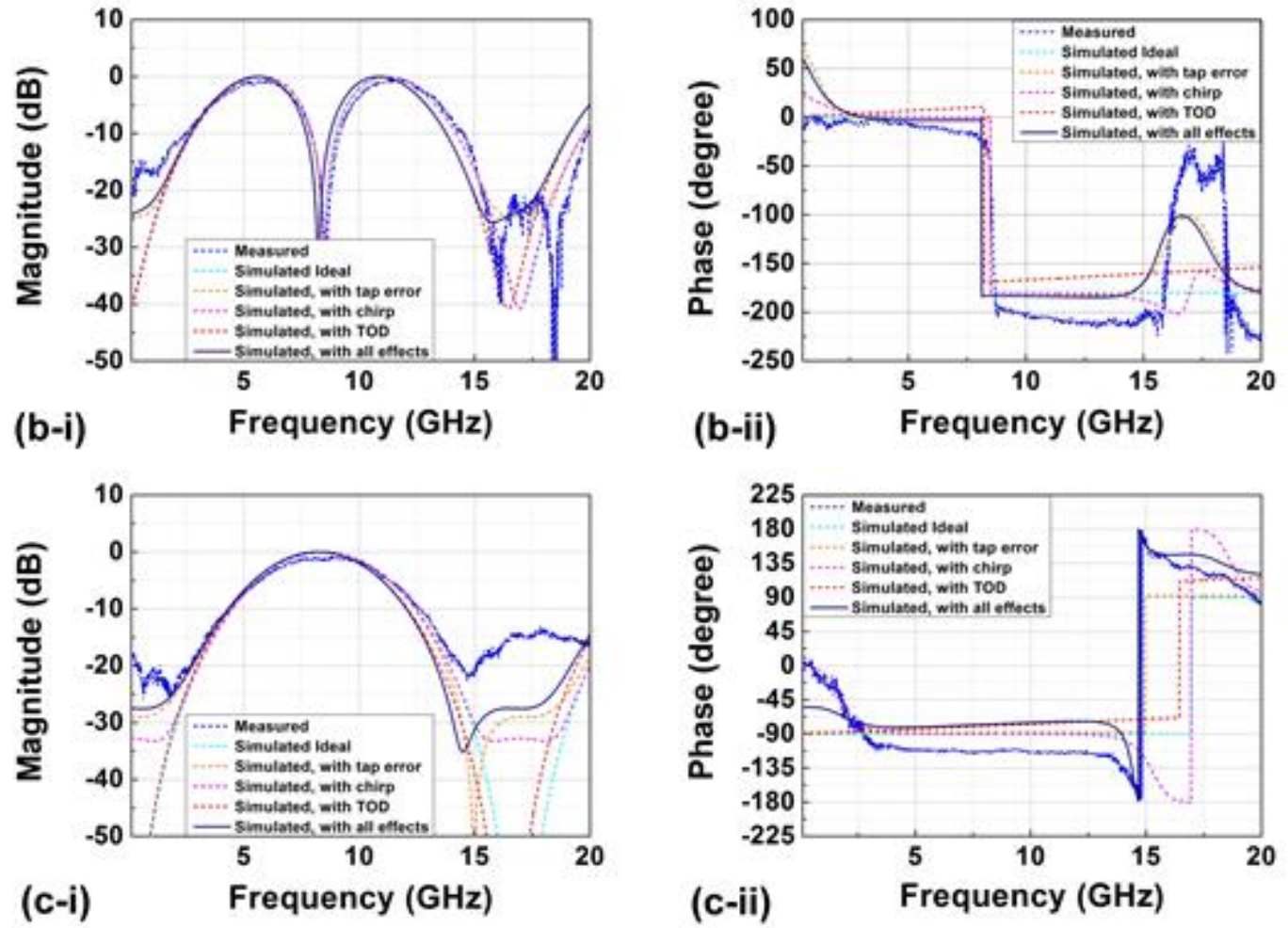

Fig. 12. Measured and simulated RF amplitude and phase responses of (a-i)-(a-ii) the first-order, (b-i)(b-ii) second-order, and (c-i)-(c-ii) third-order intensity differentiators. 

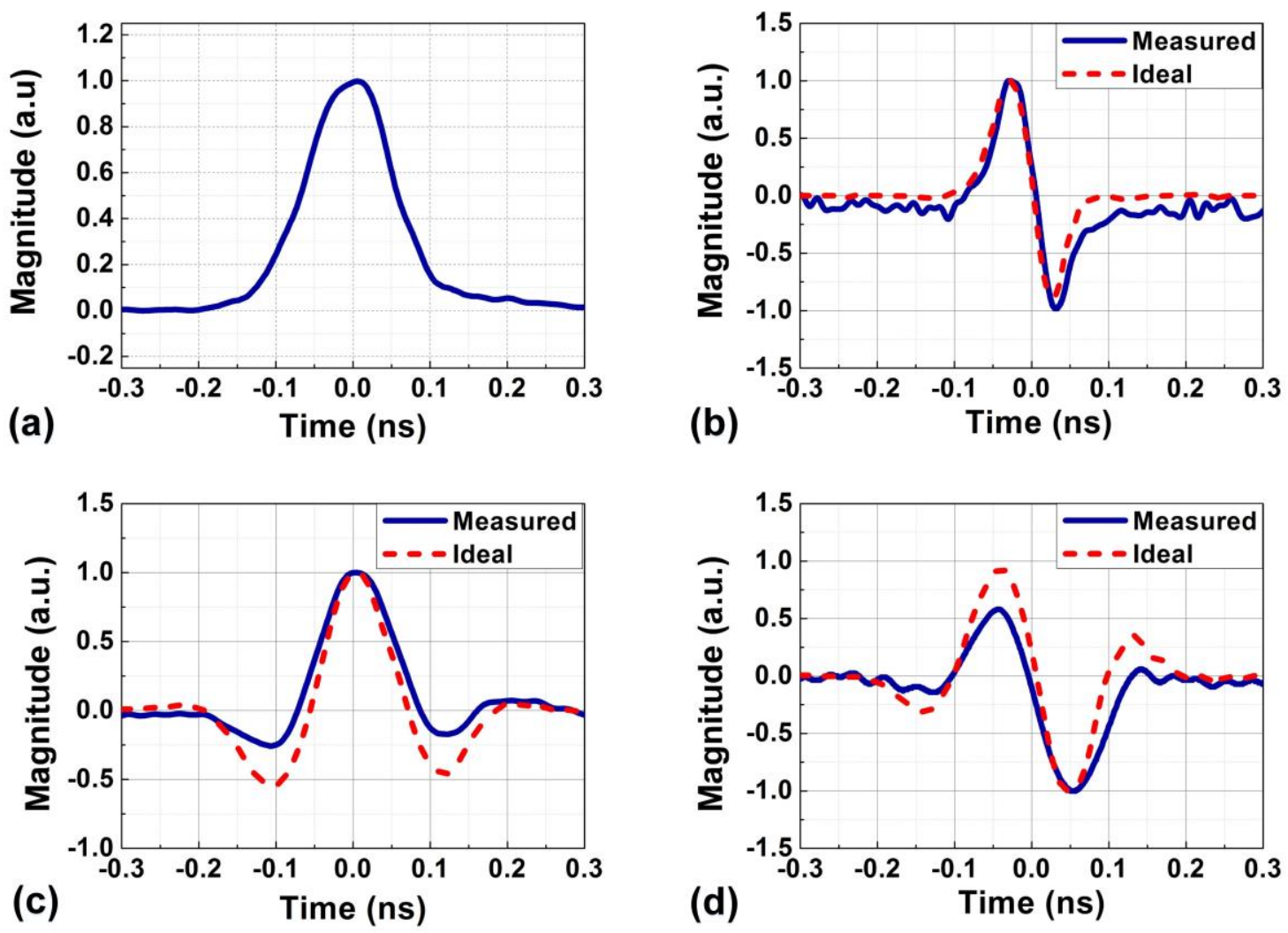

Fig.13. (a) Measured temporal waveforms of a Gaussian input pulse. Theoretical (red dashed) and experimental (blue solid) responses of the (b) first-, (b) second-, and (c) third-order differentiators.

Figure 13 


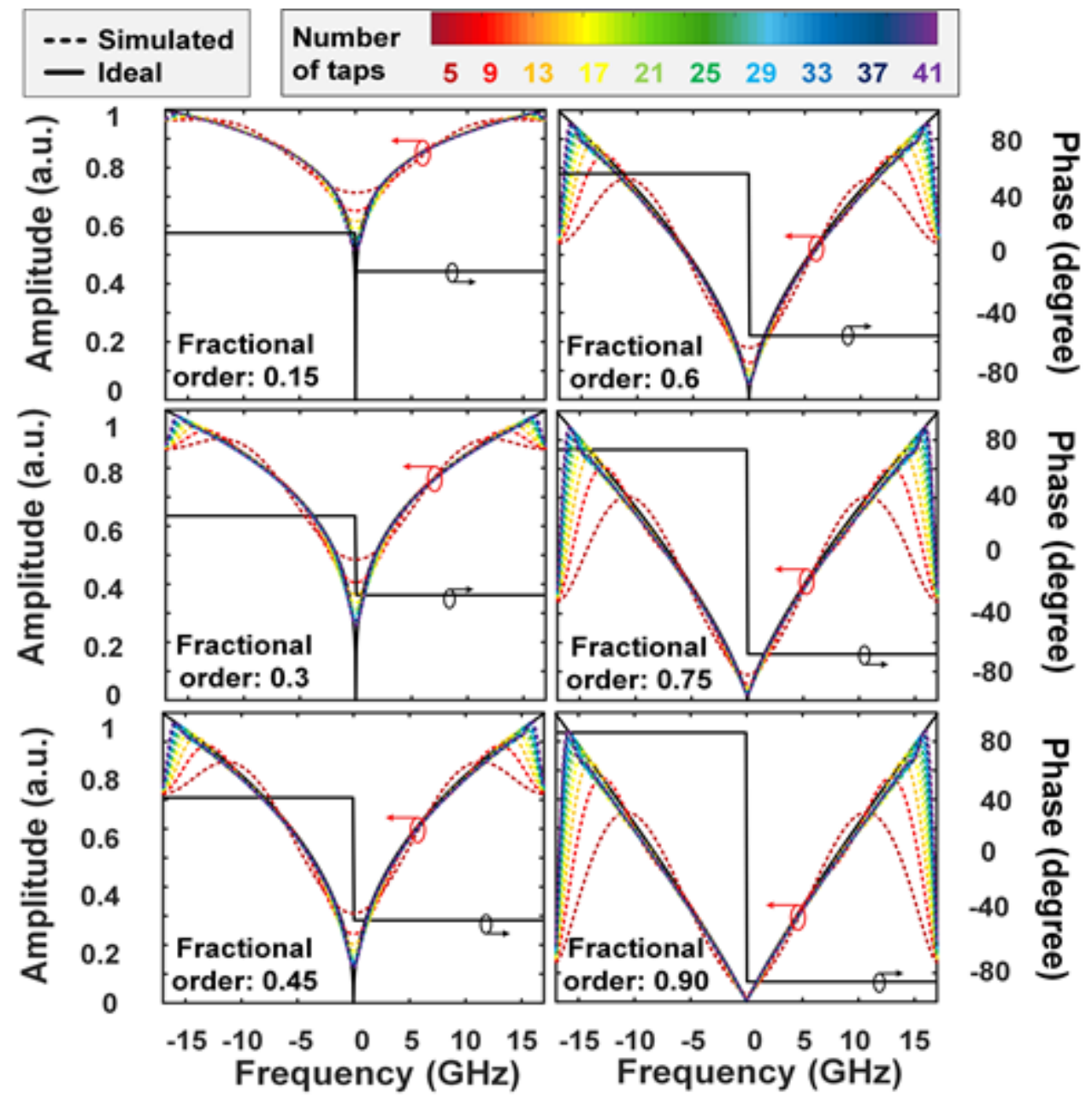

Fig. 14. Simulated transfer function of different fractional differentiation orders with varying number of taps.

Figure 14 

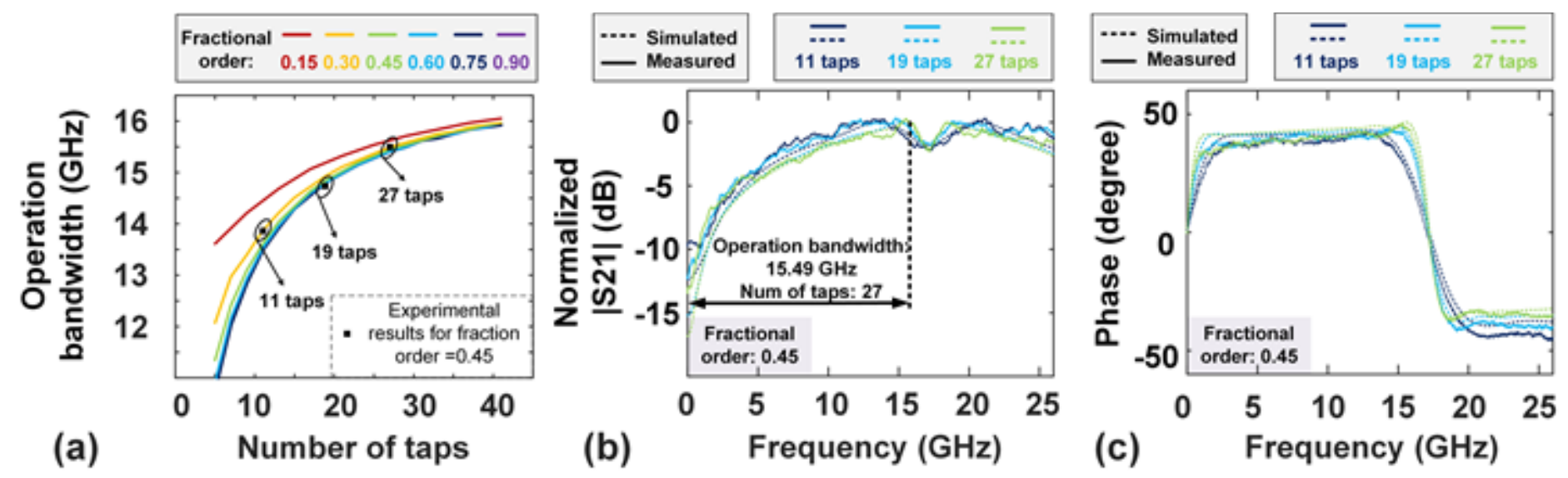

Fig. 15. (a) Relationship between the number of taps and operation bandwidth. (b, c) Experimentally demonstrated fractional differentiator with varying number of taps.

\section{Figure 15}




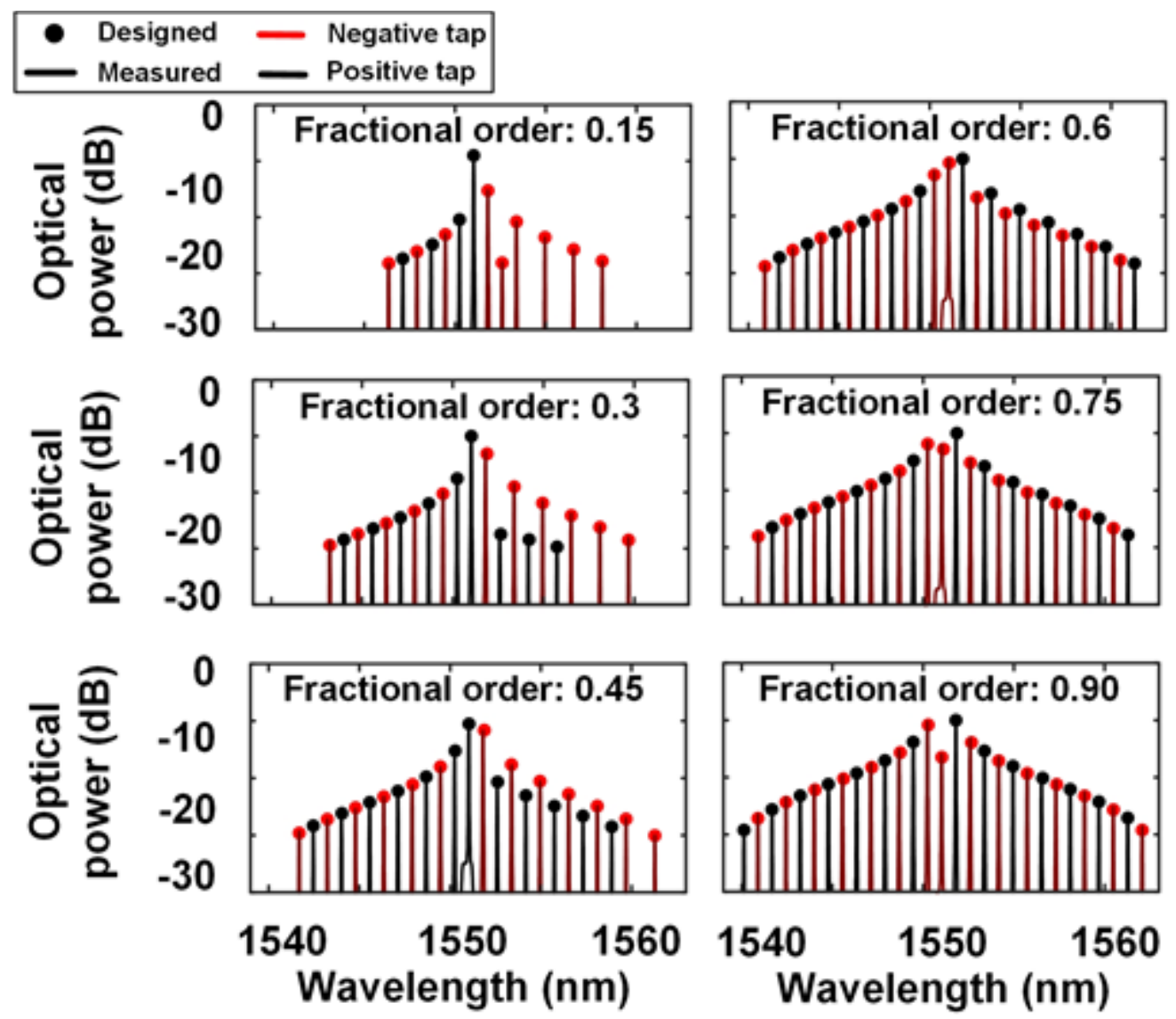

Fig. 16. Optical spectra of the shaped micro-comb for different fractional orders.

Figure 16 


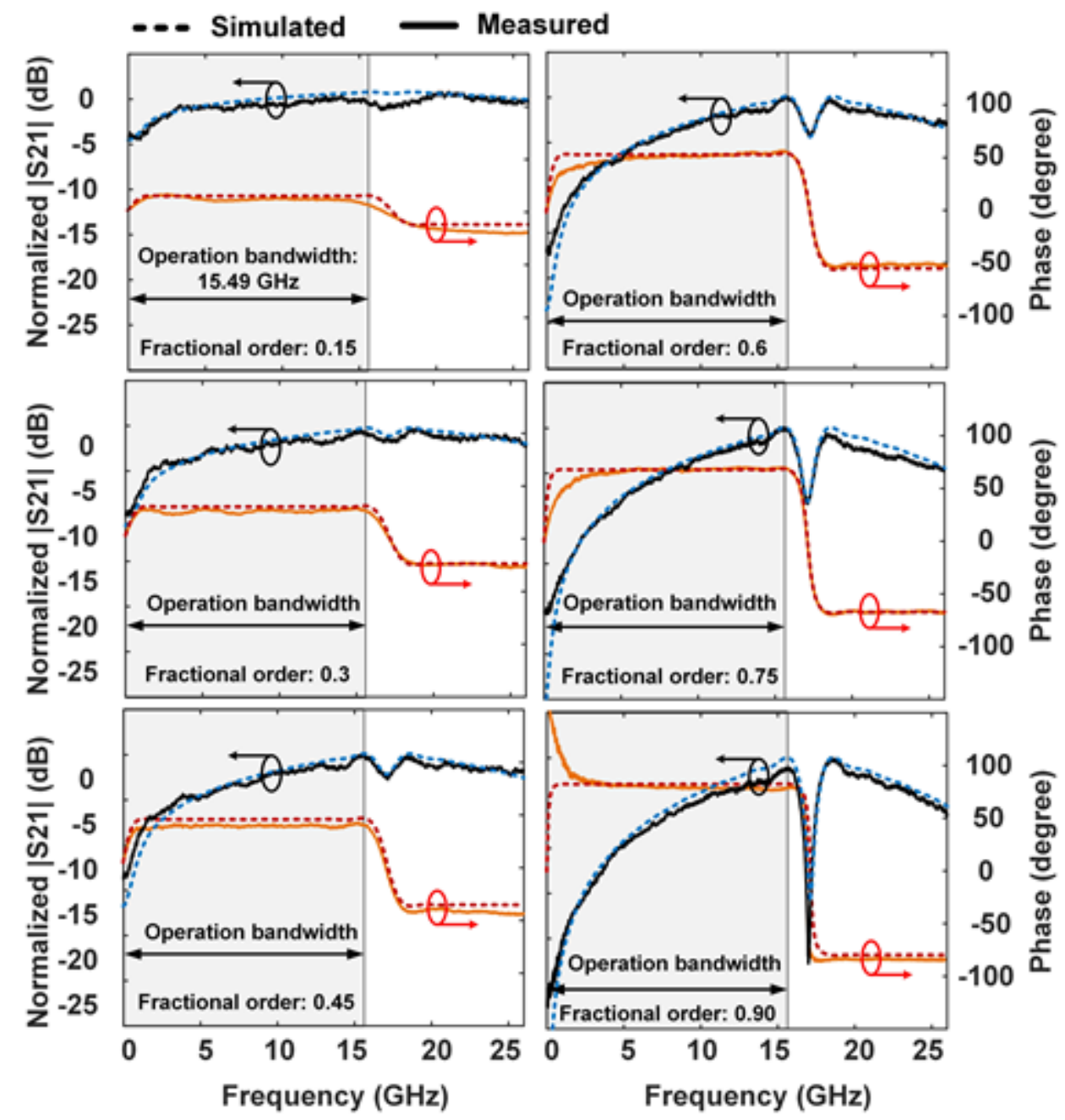

Fig. 17. Simulated and measured the transmission response of the fractional differentiator at different orders ranging from 0.15 to 0.90 . 

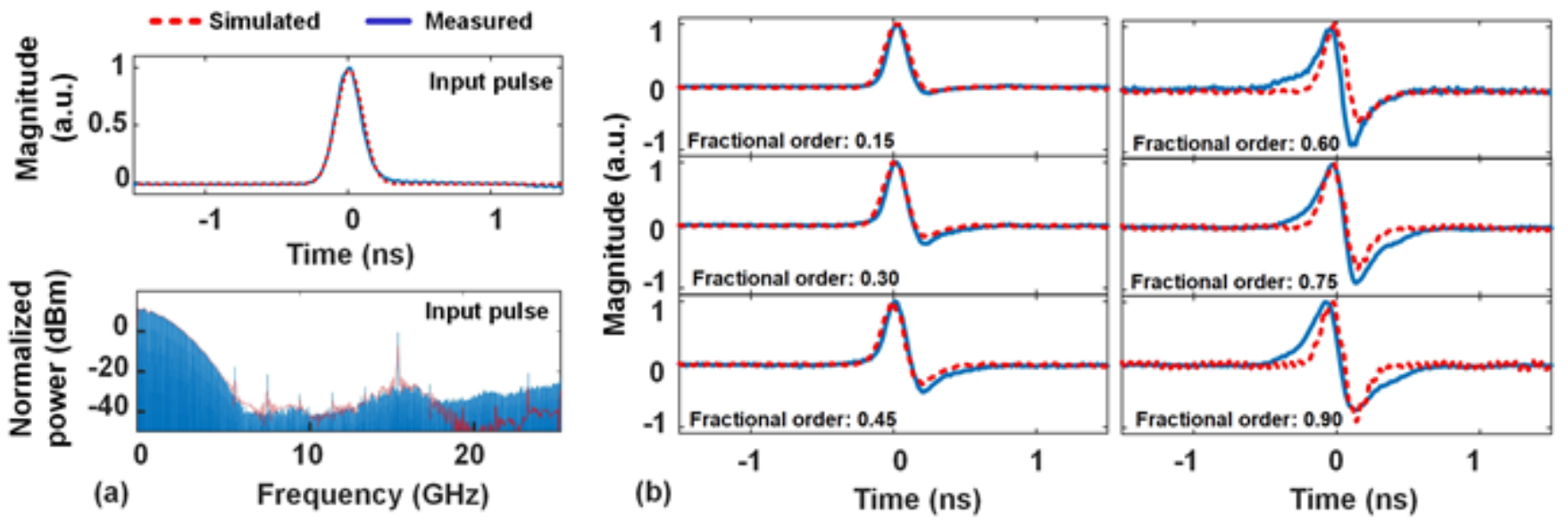

Fig. 18. Simulated and measured RF Gaussian pulse output temporal intensity waveform after the fractional differentiator.

Figure 18 


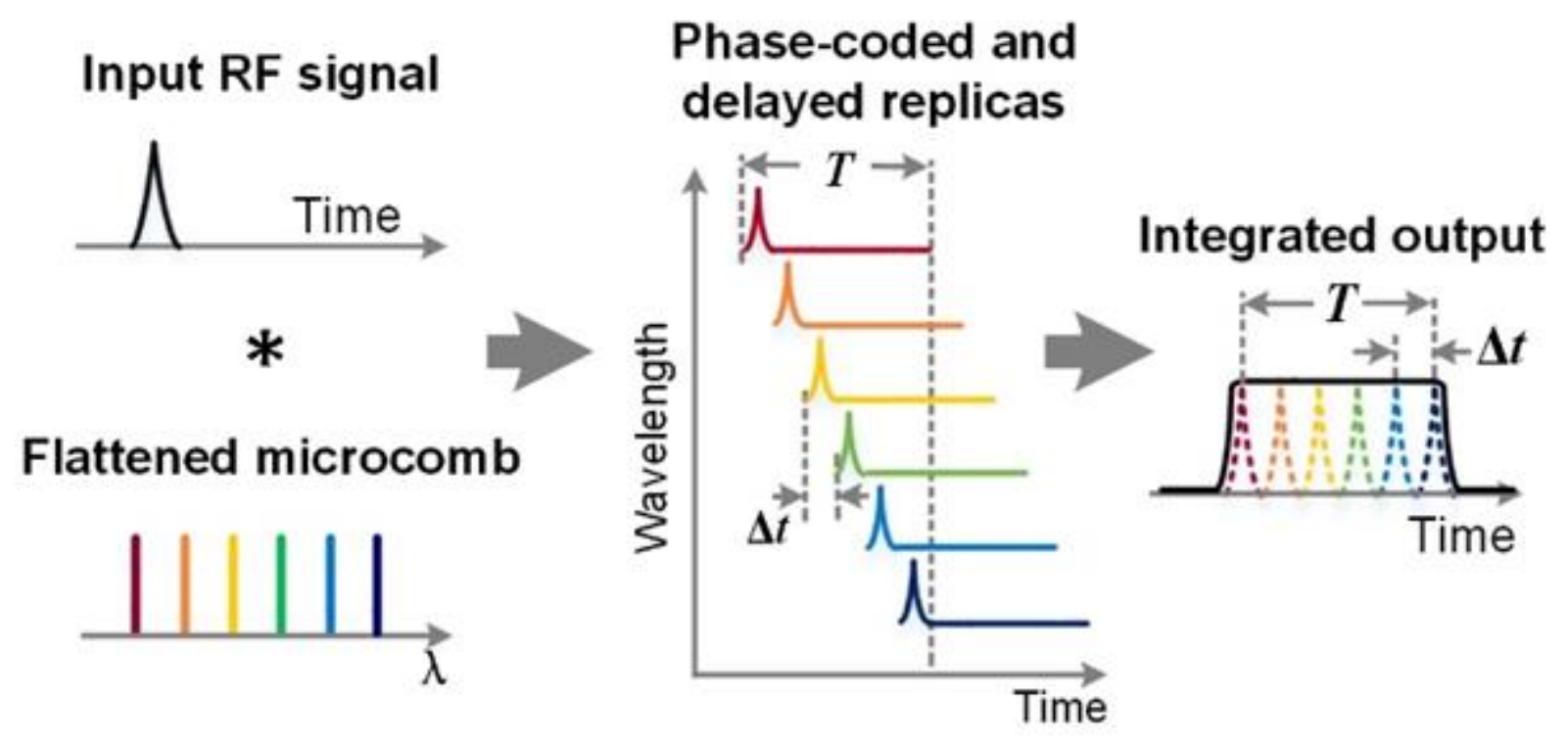

Fig. 19. Schematic diagram of the photonic RF integration.

Figure 19 

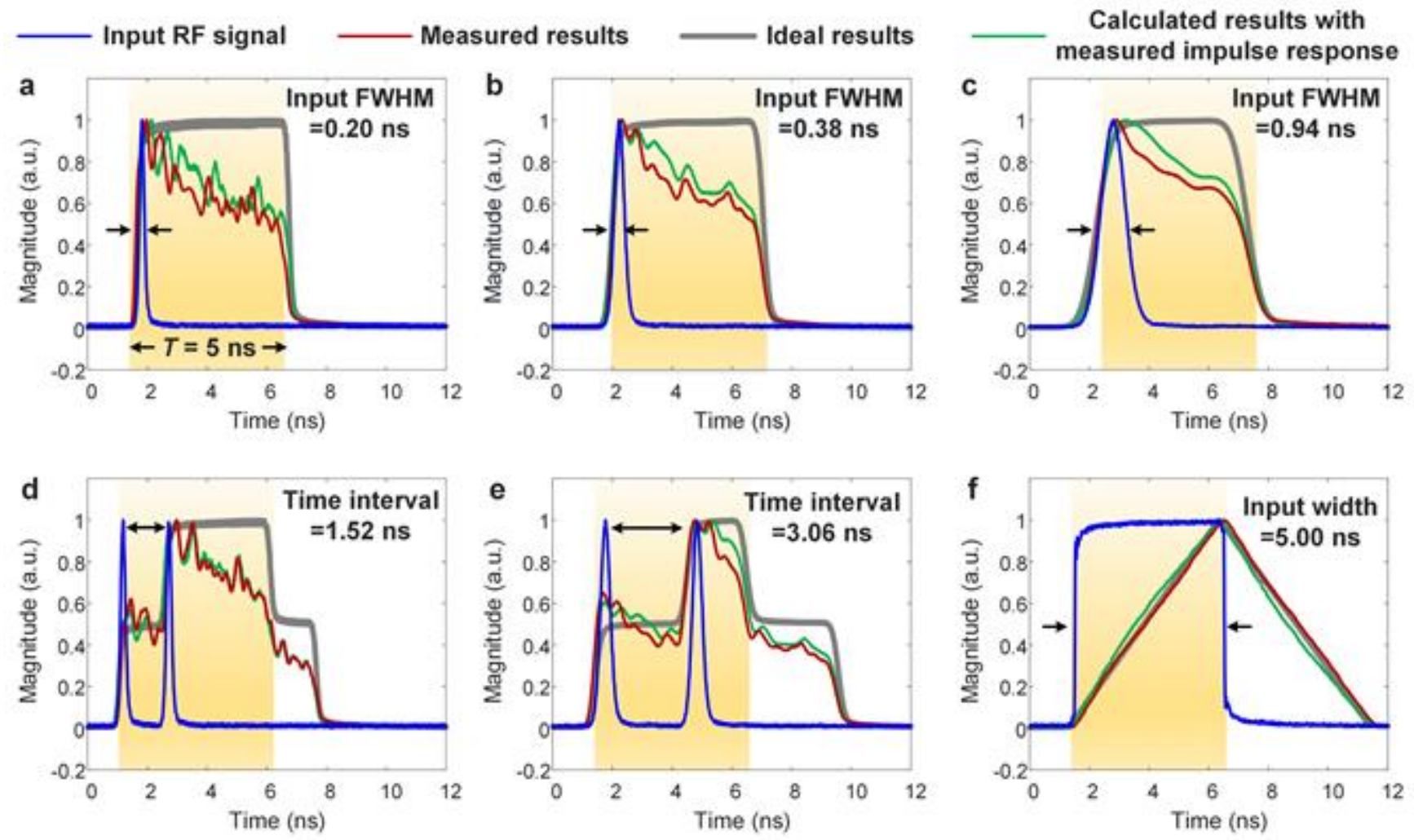

Fig. 20. Experimental results of the micro-comb-based RF integrator after comb optical power shaping for input (a-c) Gaussian pulses with FWHM of $0.20,0.38$ and $0.94 \mathrm{~ns}$, (d-e) dual Gaussian pulses with time intervals of 1.52 and $30.6 \mathrm{ns,} \mathrm{and} \mathrm{(f)} \mathrm{a} \mathrm{triangular}$ waveform with a width of $5.00 \mathrm{~ns}$. The blue curves denote the input signal, the red curves denote the measured integration results, the gray curves denote the ideal integration results, and the green curves denote the integration results calculated with the measured impulse response of the system. 

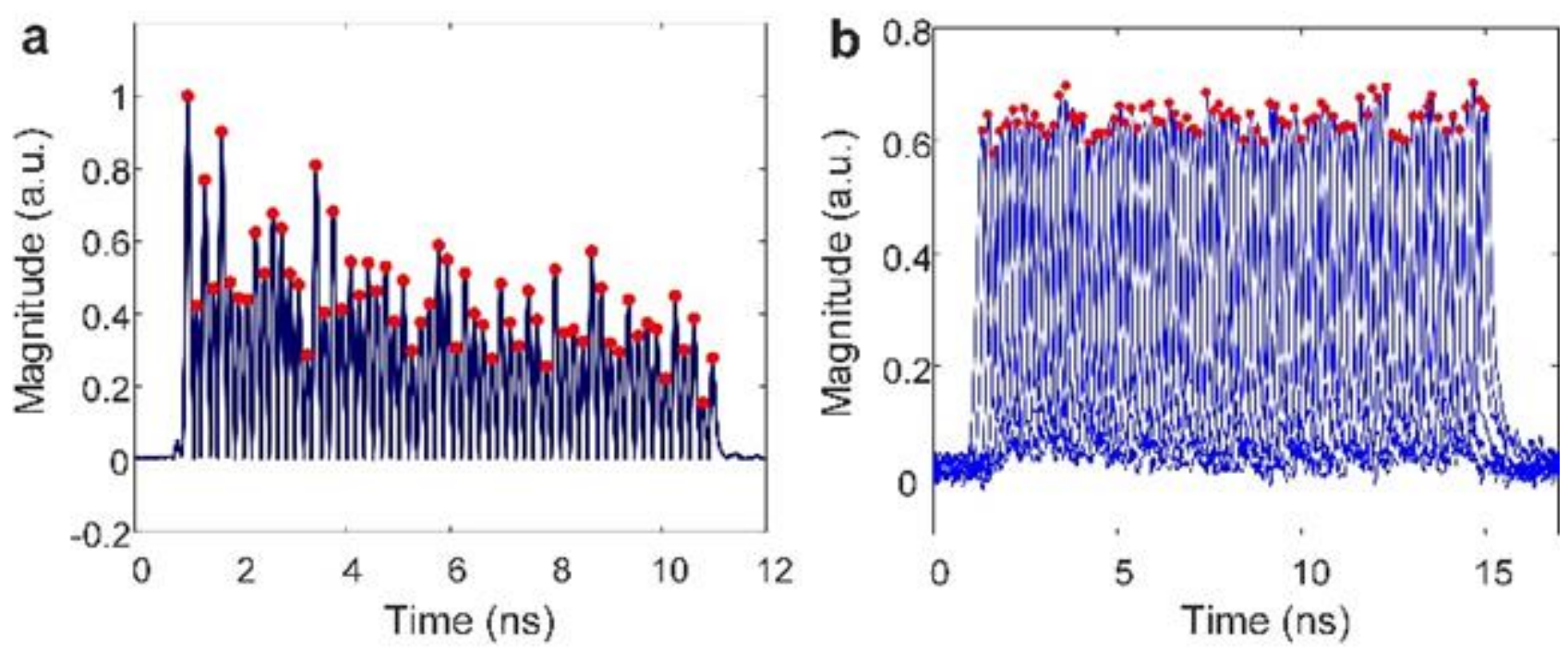

Fig. 21. Measured impulse response of the integrator (a) after comb optical power shaping and (b) after impulse response shaping using a Gaussian RF input pulse. 


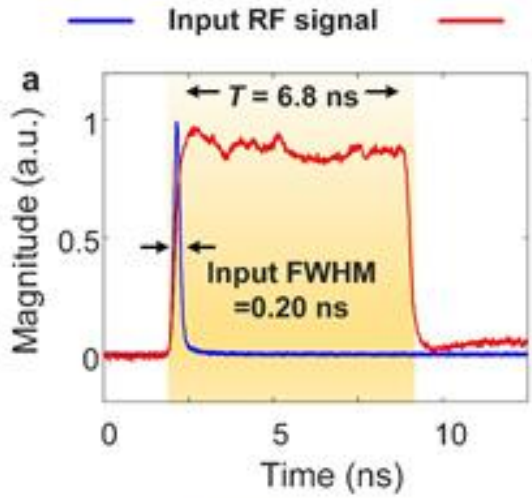

Measured integration results
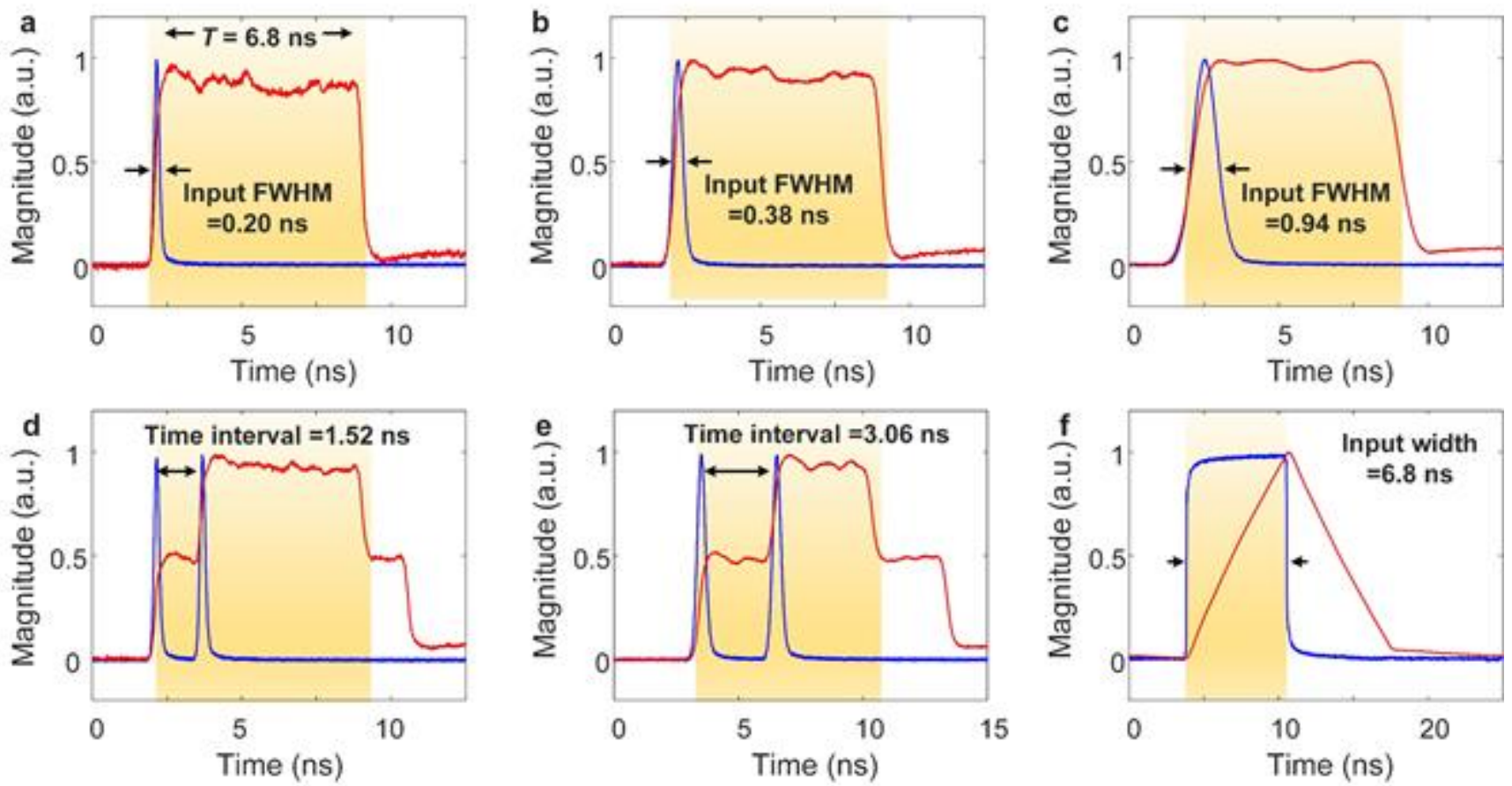

Fig. 22. Experimental results of the micro-comb-based RF integrator after impulse response shaping for input (a-c) Gaussian pulses with FWHM of $0.20,0.38$ and $0.94 \mathrm{~ns}$, (d-e) dual Gaussian pulses with time intervals of 1.52 and $3.06 \mathrm{~ns}$, and (f) a triangular waveform with a width of $5.00 \mathrm{~ns}$. The blue curves denote the input signal, the red curves denote the measured integration results. 